LBL-29824

UCB-PTH-90/48

December 1990

\title{
Exact C-Function and C-Theorem on Affine-Virasoro Space *
}

\author{
A. Giveon ${ }^{\dagger}$, M. B. Halpern, E. B. Kiritsis ${ }^{\ddagger}$, N. A. Obers ${ }^{\S}$ \\ Department of Physics, University of California \\ and \\ Theoretical Physics Group, Lawrence Berkeley Laboratory \\ Berkeley, California 94720
}

\begin{abstract}
An exact C-function, which is an action for the Virasoro master equation, is obtained on affine-Virasoro space. The solutions of the master equation are fixed points of an associated flow, which obeys a C-theorem. The closed sub-flow $S O(n)_{\text {diag }}$ is a flow on the space of graphs, and the associated Morse polynomials are known generating functions in graph theory. The general flow also implies a method for prediction of sporadic conformal deformations. We note the resemblance of this system to the expected form of an exact renormalization group equation.
\end{abstract}

Published in Nucl. Phys. B357 (1991) 655.

*This work was supported in part by the Director, Office of Energy Research, Office of High Energy and Nuclear Physics, Division of High Energy Physics of the U.S. Department of Energy under Contract DE-AC03-76SF00098 and in part by the National Science Foundation under grant PHY85-15857.The work of A.G. is supported in part by a Chaim Weizmann fellowship.

†e-mail: GIVEON@LBL.bitnet, THEORY::GIVEON, GIVEON@CSA3.LBL. Gov

$\ddagger$ e-mail: KIRITSIS@LBL.bitnet, THEORY::KIRITSIS, KIRITSIS@CSA3.LBL.Gov

§e-mail: OBERS@LBL. bitnet, THEORY::OBERS, OBERS@CSA3.LBL.Gov 


\section{Introduction}

Affine Lie algebra, or current algebra on $S_{1}$, was discovered independently in mathematics [1] and physics [2]. The first representations [2] were obtained with world-sheet fermions $[2,3]$ in the construction of current-algebraic spin and internal symmetry on the string [2]. Examples of affine-Sugawara constructions [2,4] and coset constructions [2, 4] were also given in the first string era, as well as the vertex operator construction of fermions and $S U(n)_{1}$ from compactified spatial dimensions $[5,6]$. The generalization of these constructions $[7,8,9]$ and their application to the heterotic string [10] mark the beginning of the present era. See $[11,12,13,14]$ for further historical remarks on affine-Virasoro constructions.

The general Virasoro construction on the currents $J_{a}$ of affine $g[15,16,17]$

$$
T(L)=L^{a b}{ }_{*}^{*} J_{a} J_{b} *
$$

systematizes the direct approach used by Bardakci and Halpern [2, 4] to obtain the original affine-Sugawara and coset constructions*. The Virasoro master equation $[15,16$, 17] for the inverse inertia tensor $L^{a b}=L^{b a}$ contains the affine-Sugawara nests ${ }^{\dagger}$ and many new conformal constructions $g^{\#}$ on the currents of affine $g$.

In particular, broad classes of exact solutions with unitary irrational central charge [19] have recently been obtained on affine compact $g$. The growing list presently includes the unitary irrational constructions [19, 21, 22, 23, 24]

$$
\begin{gathered}
\left(\left(\text { simply-laced } g_{x}\right)^{q}\right)_{M}^{\#} \\
S U(3)_{B A S I C}^{\#}=\left\{\begin{array}{c}
S U(3)_{M}^{\#} \\
S U(3)_{D(1)}^{\#}, \quad S U(3)_{D(2)}^{\#}, \quad S U(3)_{D(3)}^{\#} \\
S U(3)_{A(1)}^{\#}, \quad S U(3)_{A(2)}^{\#} \\
S O(2 n)^{\#}[d, 4]
\end{array}\right. \\
S O(n)_{\text {diag }}^{\#}=\left\{\begin{array}{cc}
S O(5)^{\#}[d, 2] ; & S O(2 n+1)^{\#}[d, 6]_{1}, n \geq 3 \\
S O(5)^{\#}[d, 6]_{1} ; & S O(2 n+1)^{\#}[d, 6]_{2}, n \geq 3 \\
S O(5)^{\#}[d, 6]_{2}
\end{array}\right.
\end{gathered}
$$

which are obtained for variable level in the BASIC $\supset$ Dynkin $\supset$ Maximal sequence of subansätze, and in $S O(n)_{\text {diag }}$, the diagonal ansatz on $S O(n)$. The value

$$
c\left(\left(S U(3)_{5}\right)_{D(1)}^{\#}\right)=2\left(1-\frac{1}{\sqrt{61}}\right) \simeq 1.7439
$$

*Related ideas are discussed in [18].

${ }^{\dagger}$ The affine-Sugawara nests [19] include the affine-Sugawara constructions $[2,4,8]$, the coset constructions $[2,4,9]$ and the nested coset constructions [20]. 
is the lowest unitary irrational central charge yet observed [23]. A number of unitary quadratic conformal deformations with rational central charge have also been observed, including deformations on variable level [19],

$$
\operatorname{Cartan} g^{\#}, \quad\left(S U(2)_{x} \times S U(2)_{x}\right)^{\#}, x \neq 4
$$

and the sporadic deformations $[17,24]$

$$
S U(2)_{4}^{\#}, \quad S O(2 n+1)_{2}^{\#}[d, 6], n \geq 2
$$

at levels four and two, respectively. The quadratic conformal deformations may be physically equivalent to known linear deformations [19, 25].

A very large number [19]

$$
N(g)=2^{n(g)}, \quad n(g)=\operatorname{dim} g(\operatorname{dim} g-1) / 2
$$

of solutions is expected generically on arbitrary level of any $g$, e.g. $N(g) \sim \frac{1}{4}$ billion on $S U(3)$, so the exact constructions in $(1.2)$ and $(1.4,5)$ are only the first glimpse into a generically-irrational affine-Virasoro universe of immense new structure.

Recently, a high-level or semiclassical expansion of the master equation [23] has been developed, which is capable in principle of seeing all high- $k$ smooth unitary constructions on any simple $g$. In an application to the ansatz $S O(n)_{\text {diag }}$, the expansion shows an isomorphism between this subset of affine-Virasoro constructions and the set of graphs of order $n$ [24]: We mention in particular the self-K-conjugate constructions, which are the self-complementary graphs of graph-theory [26]. These constructions occur on $S O(4 n)$ and $S O(4 n+1)$ with irrational conformal weights and half-Sugawara central charge, and are expected to occur on any simple $g$ with $\operatorname{dim} g=$ even. Conversely, the isomorphism provides a group-theoretic and conformal-field theoretic organization of graph theory, which may be interesting in mathematics.

In other directions, the master equation has been identified as an Einstein system on the group manifold [16], and a world-sheet action [27] has been obtained for the generic high-level smooth affine-Virasoro construction. Moreover, classical construction of primary fields [27] has been studied in the generic theory, and investigation of the superconformal subset of affine-Virasoro constructions has been initiated [28].

In this paper we obtain an action $A(L)$ for the Virasoro master equation, and the action is a C-function [29] on affine-Virasoro space. The solutions of the master equation, that is, the conformal field theories, are fixed points of an associated flow which satisfies a C-theorem [29]. As an example, we solve the high-level flow in $S O(n)_{\text {diag }}$, which is a flow on the space of graphs of order $n$. The associated Morse polynomials are known generating functions in graph theory. 
The general flow also implies a method for prediction of sporadic conformal deformations, which are apparently associated with collisions of level-families of conformal constructions at sporadic levels: In a simple application of the method to $S O(4)_{\text {diag }}$, we predict the deformations

$$
\begin{gathered}
S O(4)_{4}^{\#} \text { with } c=2 \\
S O(4)_{2}^{\#} \text { with } c=1, \quad S O(4) / S O(4)_{2}^{\#} \text { with } c=2 .
\end{gathered}
$$

The prediction and discovery of another deformation, $S U(3)_{3}^{\#}$ with $c=2$, will be reported elsewhere [30].

\section{The Virasoro Master Equation}

The general Virasoro construction begins with the currents $J_{a}$ of affine $g[1,2]$,

$$
\left[J_{a}^{(m)}, J_{b}^{(n)}\right]=i f_{a b}^{c} J_{c}^{(m+n)}+m G_{a b} \delta_{m+n, 0}, \quad m, n \in Z
$$

where $f_{a b}^{c}$ and $G_{a b}$ are respectively the structure constants and general Killing metric of $g$. The current algebra (2.1) is completely general since $g$ is not necessarily compact or semi-simple. In particular, to obtain level $x_{I}=2 k_{I} / \psi_{I}^{2}$ of $g_{I}$ in $g=\oplus_{I} g_{I}$ with dual Coxeter number $\tilde{h}_{I}=Q_{I} / \psi_{I}^{2}$, take

$$
G_{a b}=\oplus_{I} k_{I} \eta_{a b}^{I}, f_{a c}^{d} f_{b d}^{c}=-\oplus_{I} Q_{I} \eta_{a b}^{I}
$$

where $\eta_{a b}^{I}$ and $\psi_{I}$ are respectively the Killing metric and highest root of $g_{I}$. The class of operators quadratic in the currents,

$$
T(L) \equiv L_{*}^{a b}{ }_{*}^{*} J_{a} J_{b} * \equiv \sum_{m \in Z} L^{(m)} z^{-m-2}
$$

is defined with symmetric normal ordering, $T_{a b} \equiv{ }_{*}^{*} J_{a} J_{b}{ }_{*}^{*}=T_{b a}[15]$. The set of coefficients $L^{a b}=L^{b a}$ is called the inverse inertia tensor, in analogy with the spinning top, and the set of all coefficients $\left\{L^{a b}\right\}$ is affine-Virasoro space ${ }^{\ddagger}$. The requirement that $T(L)$ is a Virasoro operator

$$
\left[L^{(m)}, L^{(n)}\right]=(m-n) L^{(m+n)}+\frac{c}{12} m\left(m^{2}-1\right) \delta_{m+n, 0}
$$

restricts the values of $L^{a b}$ to the solutions $L_{*}^{a b}$ of the Virasoro master equation.

The master equation and central charge may be written as $[15,17]$

$$
\beta^{a b}\left(L_{*}\right)=0 \quad, \quad c\left(L_{*}\right)=2 L_{*}^{a b} P_{a b, c d} L_{*}^{c d}=2 G_{a b} L_{*}^{a b}
$$

\footnotetext{
${ }^{\ddagger}$ In a slight abuse of previous terminology, affine-Virasoro space is used here to denote the set of all inverse inertia tensors $\left\{L^{a b}\right\}$ in the quadratic form $L^{a b}{ }_{*}^{*} J_{a} J_{b}{ }_{*}^{*}$, whether the operator is Virasoro or not.
} 
where, following Ref.[15], we have defined the quantities ${ }^{\S}$

$$
\begin{aligned}
& \beta^{a b}(L) \equiv \frac{1}{2} L^{c d} L^{e f} R_{c d, e f}{ }^{a b}-L^{a b} \\
& =-L^{a b}+2 L^{a c} G_{c d} L^{d b}-L^{c d} L^{e f} f_{c e}{ }^{a} f_{d f}^{b}-L^{c d} f_{c e}{ }^{f} f_{d f}{ }^{(a} L^{b) e} \\
& P_{a b, c d} \equiv\left\langle 0\left|T_{a b}^{(2)} T_{c d}^{(-2)}\right| 0\right\rangle=G_{a(c} G_{d) b}-\frac{1}{2} f_{a(c}{ }^{e} f_{d) b}{ }^{f} G_{e f} .
\end{aligned}
$$

For affine compact $g$, the matrix $P_{a b, c d}$ is non-negative when each level $x_{I}$ of $g_{I}$ is some positive integer, and $P_{a b, c d}$ is also invertible when each integer $x_{I}>1$. The explicit form of the tensor $R_{c d, e f}^{a b}=R_{e f, c d}^{a b}$ is given in [15], and some of its properties are collected in Appendix A. In particular, the two forms of the central charge in (2.5) are related by the master equation $\beta^{a b}\left(L_{*}\right)=0$ and the first identity in eq.(A.20). In what follows, we refer to the quantity $\beta^{a b}(L)$ in (2.6a) as the $\beta$-function.

The master equation has been identified in [16] as an Einstein-like system on the group manifold: The central charge of the general construction is $c=\operatorname{dim} g-4 R$, where $R$ is the Einstein curvature scalar.

We remark on some general properties of the master equation which will be useful in the analysis below.

1. The affine-Sugawara construction $[2,4,8] L_{g}$ is

$$
L_{g}^{a b}=\oplus_{I} \frac{\eta_{I}^{a b}}{2 k_{I}+Q_{I}}, \quad c_{g}=\sum_{I} \frac{x_{I} \operatorname{dim} g_{I}}{x_{I}+\tilde{h}_{I}}
$$

for arbitrary level of any $g$, and similarly for $L_{h}$ when $h \subset g$.

2. K-conjugation covariance $[2,4,9,15]$. When $L_{*}$ is a solution of the master equation on $g$, then so is the K-conjugate partner $\tilde{L}_{*}$ of $L_{*}$

$$
\tilde{L}_{*}^{a b}=L_{g}^{a b}-L_{*}^{a b}, \quad, \quad c\left(\tilde{L}_{*}\right)=c_{g}-c\left(L_{*}\right)
$$

while the corresponding stress tensors $T\left(L_{*}\right)$ and $T\left(\tilde{L}_{*}\right)$ form a commuting pair of Virasoro operators. Repeated embedding by K-conjugation generates the coset constructions $[2,4,9]$, the nested coset constructions or affine-Sugawara nests [20, 19] and the affineVirasoro nests [19].

3. Unitarity $[9,19]$. Unitary constructions on positive integer level of affine compact $g$ are recognized when $L_{*}^{a b}=$ real in any Cartesian basis, and corresponding forms in other bases, since $\left(J_{a}^{(n)}\right)^{\dagger}=J_{a}^{(-n)}$ implies $\left(L^{(n)}\right)^{\dagger}=L^{(-n)}$. Unitarity guarantees that $c\left(L_{*}\right) \geq 0$,

${ }^{\S}$ Our notation is $A_{(a} B_{b)} \equiv A_{a} B_{b}+A_{b} B_{a}$ and $A_{[a} B_{b]} \equiv A_{a} B_{b}-A_{b} B_{a}$.

"At level two of the vacuum module of affine compact $g$, the null states at integer $x>1$ are constructed by application of non-positively moded currents on the states $E_{\alpha}^{(0)}|0\rangle$, where $\alpha$ is a simple root of $g$ [31]; these states are not in our list $T_{a b}^{(-2)}|0\rangle=\frac{1}{2} J_{(a}^{(-1)} J_{b)}^{(-1)}|0\rangle$, and the argument is easily extended to semi-simple $g$. 
and K-conjugate partners of unitary constructions are also unitary with $c\left(\tilde{L}_{*}\right) \geq 0$. The double inequality

$$
0 \leq c\left(L_{*}\right) \leq c_{g}
$$

follows for all unitary Virasoro constructions on affine compact $g$. Correspondingly, all unitary high-level central charges on simple compact $g$ are integer valued from 0 to $\operatorname{dim} g[21,23]$. It is also known [9] that all unitary Virasoro constructions satisfying $0 \leq c\left(L_{*}\right)<1$ can be realized as coset constructions $g / h$. It follows that all unitary affine-Virasoro constructions satisfying $c_{g}-1<c\left(L_{*}\right) \leq c_{g}$ are realizable as the Kconjugate partners $h \subset g$ of the coset constructions between 0 and 1. Moreover, all unitary solutions with high-level central charge $0,1, \operatorname{dim} g-1$ and $\operatorname{dim} g$ are known for simple compact $g[23]$.

4. Automorphisms $[21,23,24]$. The inverse inertia tensor transforms under the inner and outer automorphisms of $g$ as

$$
\left(L^{\prime}\right)^{a b}=L^{c d}\left(\omega^{-1}\right)_{c}^{a}\left(\omega^{-1}\right)_{d}^{b}, \omega \in \operatorname{Aut}(g) .
$$

It follows from the definition of the $\beta$-function in (2.6a) that

$$
\beta^{a b}\left(L^{\prime}\right)=\beta^{c d}(L)\left(\omega^{-1}\right)_{c}^{a}\left(\omega^{-1}\right)_{d}^{b}
$$

so $\left(L_{*}^{\prime}\right)^{a b}$ is an automorphically equivalent solution of the master equation when $L_{*}^{a b}$ is a solution.

5. Self K-conjugate constructions [24]. A large number of self K-conjugate constructions

$$
\tilde{L}_{*}^{a b}=L_{*}^{c d}\left(\omega^{-1}\right)_{c}{ }^{a}\left(\omega^{-1}\right)_{d}^{b} \quad, \quad \text { for some } \omega \in \operatorname{Aut}(g)
$$

has been observed on $S O(4 n)$ and $S O(4 n+1)$, which correspond to the self-complementary graphs of graph theory. These constructions exhibit irrational conformal weights and halfSugawara central charge $c\left(L_{*}\right)=c_{g} / 2$, and are expected to occur on any simple group with $\operatorname{dim} g=$ even.

\section{The C-Function and a C-Theorem}

As seen in eqs. (2.5-6), the basic tensors of the master equation and central charge are $R_{a b, c d}^{e f}=R_{c d, a b}{ }^{e f}$ and $P_{a b, c d}=P_{c d, a b}$, and it is clear from its definition that $P_{a b, c d}$ is a natural metric ${ }^{\|}$on affine-Virasoro space $\left\{L^{a b}\right\}$. In fact, as noted in Appendix A, the $R$ tensor with all indices covariant

$$
R_{a b, c d, e f} \equiv R_{a b, c d}^{g h} P_{g h, e f}
$$

\footnotetext{
${ }^{\|}$Reparametrization covariant structures on affine-Virasoro space are discussed in Appendix B.
} 
is a completely symmetric tensor. On the basis of this observation, we construct the following Lagrangian for the master equation

$$
\begin{aligned}
A(L) & \equiv 6 L^{a b} L^{c d} P_{a b, c d}-2 L^{a b} L^{c d} L^{e f} R_{a b, c d, e f} \\
& =2 L^{a b} P_{a b, c d}\left(L^{c d}-2 \beta^{c d}(L)\right) .
\end{aligned}
$$

It is easily checked that

$$
\beta^{a b}(L)=-\frac{1}{12} P^{a b, c d} \frac{\partial A(L)}{\partial L^{c d}}
$$

where

$$
P^{a b, e f} P_{e f, c d}=\frac{1}{2} \delta_{(c}^{a} \delta_{d)}^{b} \equiv \delta_{c d}^{a b}, \frac{\partial L^{a b}}{\partial L^{c d}}=\delta_{c d}^{a b}
$$

so the master equation $\beta^{a b}\left(L_{*}\right)=0$ is the equation of motion of $A(L)$ when the metric $P_{a b, c d}$ is invertible.

The Lagrangian $A(L)$ is in fact a C-function [29] on affine-Virasoro space, since

$$
A\left(L_{*}\right)=c\left(L_{*}\right)
$$

follows from the master equation, the definition (3.2) and the forms of the central charge in $(2.5)$.

The symmetries of the C-function include the automorphisms of $g$

$$
A\left(L^{\prime}\right)=A(L), L^{\prime}=\omega L \omega^{-1}, \omega \in \operatorname{Aut}(g)
$$

and accidental symmetries which correspond to sporadic conformal deformations [17, 24], as we will remark below. The operation of K-conjugation is defined for any point $L$ in affine-Virasoro space as $\tilde{L} \equiv L_{g}-L$ where $L_{g}$ is the affine-Sugawara construction on $g$. The corresponding transformation of the action and its derivatives

$$
\begin{gathered}
A(\tilde{L})=c_{g}-A(L) \\
\frac{\partial A(\tilde{L})}{\partial \tilde{L}^{a b}}=\frac{\partial A(L)}{\partial L^{a b}} \Rightarrow \beta^{a b}(\tilde{L})=\beta^{a b}(L) \\
\frac{\partial^{2} A(\tilde{L})}{\partial \tilde{L}^{a b} \partial \tilde{L}^{c d}}=-\frac{\partial^{2} A(L)}{\partial L^{a b} \partial L^{c d}}
\end{gathered}
$$

are easily verified with the second identity in (A.20). Eqs. (3.7) show that K-conjugation is not a symmetry of the C-function but it is a covariance of the equations of motion, $\beta^{a b}\left(L_{*}\right)=0$, as expected. Such "quasi-symmetries" of the action are familiar in physics, leading e.g. to WZW level quantization.

We remark for use below that the Laplacian of the C-function has the simple form

$$
\Delta A(L) \equiv P^{a b, c d} \frac{\partial^{2} A(L)}{\partial L^{a b} \partial L^{c d}}=-12 \frac{\partial \beta^{a b}(L)}{\partial L^{a b}}
$$




$$
=12(\operatorname{dim} g+1)\left(\frac{1}{2} \operatorname{dim} g-L_{a b}^{g} L^{a b}\right)
$$

where

$$
L_{a b}^{g}=2 G_{a b}-f_{a c}^{d} f_{b d}^{c}
$$

is the inverse of the affine-Sugawara construction. The even simpler result

$$
\Delta A\left(L_{*}\right)=-12 \frac{\operatorname{dim} g(\operatorname{dim} g+1)}{c_{g}}\left[c\left(L_{*}\right)-\frac{c_{g}}{2}\right]
$$

is obtained with eq. (2.5) at any fixed point $L_{*}$ on simple $g$.

It is natural to define an associated flow on affine-Virasoro space

$$
\dot{L}^{a b} \equiv \frac{d L^{a b}}{d t}=\beta^{a b}(L)=-\frac{1}{12} P^{a b, c d} \frac{\partial A(L)}{\partial L^{c d}}
$$

although the physical interpretation of the time variable $t$, and in particular the connection to exact renormalization group equations, has not yet been understood. It is clear that all the solutions of the master equation are fixed points of the flow

$$
\left.\dot{L}^{a b}\right|_{L=L_{*}}=0 \quad, \quad \beta^{a b}\left(L_{*}\right)=0
$$

and moreover that the flow automatically satisfies the identity

$$
\dot{A}(L)=-\frac{1}{12} \frac{\partial A}{\partial L^{a b}} P^{a b, c d} \frac{\partial A}{\partial L^{c d}}=-12 \beta^{a b}(L) P_{a b, c d} \beta^{c d}(L) .
$$

The C-theorem

$$
\dot{A}(L) \leq 0
$$

follows for the flow on positive integer levels of affine compact $g$, since $P_{a b, c d}$ is a nonnegative matrix in this case.

\section{The Flow Near a Fixed Point}

In order to study the flow (3.11) on affine-Virasoro space, it is convenient to define its stability matrix $\mathcal{S}$

$$
\begin{aligned}
\mathcal{S}^{a b}{ }_{c d}(L) & \equiv \frac{1}{12} P^{a b, e f} \frac{\partial^{2} A(L)}{\partial L^{e f} \partial L^{c d}}=-\frac{\partial \beta^{a b}(L)}{\partial L^{c d}} \\
& =\delta_{c d}^{a b}-L^{e f} R_{e f, c d}^{a b}
\end{aligned}
$$

which controls the flow near a fixed point $L=L_{*}+\delta L$ according to

$$
\frac{d}{d t} \delta L^{a b}=-\mathcal{S}^{a b}{ }_{c d}\left(L_{*}\right) \delta L^{c d}
$$


when $\delta L$ is small. To organize the problem, we introduce the left and right eigenvectors of the stability matrix

$$
\psi_{a b}^{i}(L) \mathcal{S}^{a b}{ }_{c d}(L)=s_{i}(L) \psi_{c d}^{i}(L) \quad, \quad \mathcal{S}^{a b}{ }_{c d}(L) \chi_{i}^{c d}(L)=s_{i}(L) \chi_{i}^{a b}(L)
$$

where $i=1,2, \cdots, \operatorname{dim} g(\operatorname{dim} g+1) / 2$. It is easy to see from the definition (4.3) that we may require

$$
\psi_{a b}^{i}=P_{a b, c d} \chi_{i}^{c d}
$$

among the left and right eigenvectors. Choosing orthogonality in degenerate subspaces, we also obtain the statements of orthonormality and completeness,

$$
\begin{gathered}
\psi_{a b}^{i} \chi_{j}^{a b}=\delta_{j}^{i} \Rightarrow \psi_{a b}^{i} P^{a b, c d} \psi_{c d}^{j}=\chi_{i}^{a b} P_{a b, c d} \chi_{j}^{c d}=\delta^{i j} \\
\sum_{i} \psi_{a b}^{i} \psi_{c d}^{i}=P_{a b, c d} \quad, \quad \sum_{i} \chi_{i}^{a b} \chi_{i}^{c d}=P^{a b, c d} .
\end{gathered}
$$

Moreover, we have the spectral resolution of $\mathcal{S}$

$$
\mathcal{S}^{a b}{ }_{c d}(L)=\sum_{i} \chi_{i}^{a b}(L) s_{i}(L) \psi_{c d}^{i}(L)
$$

which implies that

$$
\mathcal{S}^{a b}{ }_{a b}(L)=\frac{1}{12} \Delta A(L)=\sum_{i} s_{i}(L) .
$$

Finally, the eigenvectors $\delta L^{i}$ of the flow satisfy

$$
\begin{gathered}
\frac{d}{d t} \delta L^{i}=-s_{i} \delta L^{i} \\
\delta L^{i} \equiv \delta L^{a b} \psi_{a b}^{i}, \quad \delta L^{a b}=\sum_{i} \delta L^{i} \chi_{i}^{a b}
\end{gathered}
$$

and, in particular, the corresponding operators $\chi_{i}^{a b}\left(L_{*}\right) T_{a b}$ of the fixed point $L_{*}$ are

$$
\begin{aligned}
& \text { relevant when } s_{i}\left(L_{*}\right)<0 \\
& \text { irrelevant when } s_{i}\left(L_{*}\right)>0 \\
& \text { marginal when } s_{i}\left(L_{*}\right)=0
\end{aligned}
$$

where the categories correspond to unstable, stable and metastable directions as usual.

We list some simple properties of the eigenvalues and eigenvectors of $\mathcal{S}$ :

1. $\tilde{L}_{g}=0$ and $L_{g}$. The values of the stability matrix at $L_{*}=L_{g}$ and $\tilde{L}_{g}=0$

$$
\mathcal{S}^{a b}{ }_{c d}\left(L_{g}\right)=-\delta_{c d}^{a b}, \quad \mathcal{S}^{a b}{ }_{c d}(0)=+\delta_{c d}^{a b}
$$

are computed directly from (4.1) and the second affine-Sugawara identity in (A.20). It follows that all eigenvalues of $\mathcal{S}$ are degenerate in these cases, with values

$$
s_{i}\left(L_{g}\right)=-1, \quad s_{i}(0)=+1 .
$$


This implies in particular that all operators $\delta L^{a b} T_{a b}$ are relevant operators near the affineSugawara construction, while all operators are irrelevant at $\tilde{L}_{g}=0$.

2. K-conjugate theories. The K-conjugation identity $(3.7 \mathrm{c})$ may be written

$$
\mathcal{S}^{a b}{ }_{c d}(\tilde{L})=-\mathcal{S}^{a b}{ }_{c d}(L)
$$

It follows that

$$
\left\{s_{i}(\tilde{L})\right\}=\left\{-s_{i}(L)\right\}
$$

and we may choose

$$
\chi_{s}^{a b}(\tilde{L})=\chi_{-s}^{a b}(L)
$$

Physically, this means that a relevant operator $\chi_{i}^{a b}\left(L_{*}\right) T_{a b}$ of the fixed point $L_{*}$ is an irrelevant operator at the K-conjugate fixed point $\tilde{L}_{*}$ and vice-versa, and that every marginal operator of $L_{*}$ is also a marginal operator of $\tilde{L}_{*}$.

3. Universal \pm 1 eigenvalues. For all fixed points $L_{*}$ the relations ${ }^{* *}$

$$
\mathcal{S}^{a b}{ }_{c d}\left(L_{*}\right) L_{*}^{c d}=-L_{*}^{a b}, \quad \mathcal{S}^{a b}{ }_{c d}\left(L_{*}\right) \tilde{L}_{*}^{c d}=+\tilde{L}_{*}^{a b}
$$

are easily obtained from the definition of $\mathcal{S}$ in (4.1), the master equation (2.5-6) and the K-conjugation identity (4.11). It follows that

$$
\chi_{1}^{a b}\left(L_{*}\right)=L_{*}^{a b} \quad, \quad \chi_{2}^{a b}\left(L_{*}\right)=\tilde{L}_{*}^{a b}
$$

are right eigenvectors of $\mathcal{S}\left(L_{*}\right)$ with $s_{1}=-1$ and $s_{2}=+1$, unless $L_{*}=0$ or $\tilde{L}_{*}=0$.

4. Zero eigenvalues of $\mathcal{S}$. According to the definitions (4.1) and (4.3), any eigenvector $\chi_{0}^{a b}\left(L_{*}\right)$ with $s\left(L_{*}\right)=0$ solves the linearized master equation [19]

$$
\chi_{0}^{a b}\left(L_{*}\right)=L_{*}^{c d} \chi_{0}^{e f}\left(L_{*}\right) R_{c d, e f}^{a b}
$$

so the marginal operators $\chi_{0}^{a b}\left(L_{*}\right) T_{a b}$ of $L_{*}$ correspond to continuous solutions $L_{*}^{a b}+$ $\delta L \chi_{(0)}^{a b}\left(L_{*}\right)$ of the master equation near $L_{*}$. Among these, we must distinguish the quadratic deformations $[17,19,24,25]$ from those directions associated to the physically irrelevant orbit $\operatorname{Aut}\left(L_{*}\right)$ of $\operatorname{Aut}(g)$ on $L_{*}$.

5. Aut $\left(L_{*}\right)$. The infinitesimal automorphisms of $g$

$$
\omega_{a}^{b}=\delta_{a}^{b}+\xi^{c} f_{c a}{ }^{b}+\mathcal{O}\left(\xi^{2}\right)
$$

induce the infinitesimal transformations $\delta L=L^{\prime}-L$

$$
\delta L^{a b}(\xi)=L^{c(a} f_{c d}^{b)} \xi^{d}
$$

${ }^{* *}$ In fact, the master equation $\beta^{a b}\left(L_{*}\right)=0$ can be resolved in the eigenvalue basis $L_{*}^{a b}=\sum_{i} L_{*}^{i} \chi_{i}^{a b}\left(L_{*}\right)$ as $L_{*}^{i}\left(s_{i}\left(L_{*}\right)+1\right)=0$, which shows the usual number $2^{N(g)}, N(g)=\operatorname{dim} g(\operatorname{dim} g+1) / 2$ of generic solutions, as expected, according to $s_{i}\left(L_{*}\right)=-1$ or $L_{*}^{i}=0$ for each $i$. 
according to eq.(2.10). The corresponding finite transformation of the $\beta$-function is given in (2.11). The infinitesimal form of this relation is that the stability matrix satisfies

$$
\mathcal{S}^{a b}{ }_{c d}(L) \delta L^{c d}(\xi)=0
$$

since $\beta^{a b}(L+\delta L) \simeq \beta^{a b}(L)-\mathcal{S}^{a b}{ }_{c d}(L) \delta L^{c d}$. It follows that

$$
\chi^{a b}(L, \xi) \equiv \delta L^{a b}(\xi)=L^{c(a} f_{c d}^{b)} \xi^{d}, \quad s(\xi)=0
$$

is a set of zero-eigenvalue eigenvectors of $\mathcal{S}$, unless $\delta L(\xi)=0$ for some $\xi$. Vanishing of $\delta L(\xi)$ for some $\xi$ reflects a symmetry of $L$ under some Lie group $h \subset g$, so we find

$$
(\operatorname{dim} g-\operatorname{dim} h) \text { marginal operators } \chi^{a b}\left(L_{*}, \xi\right) T_{a b}
$$

with $\xi \in g / h$ when $L_{*}^{a b}$ is Lie $h$-symmetric. These marginal directions are not physically relevant because they follow the orbit $\operatorname{Aut}\left(L_{*}\right)$ of $A u t(g)$ on $L_{*}$, and the directions can in principle be removed by "gauge fixing". Any higher accidental symmetry of the Cfunction (3.2) will similarly produce further marginal directions of the form (4.19), which must then correspond to quadratic deformations.

6. Self K-conjugate constructions. According to the characterization of self Kconjugate constructions in (2.12) and the K-conjugation identity (4.11), the non-zero eigenvalues of a self K-conjugate construction $L_{*}$ will occur in $\pm s$ pairs, so that

$$
\begin{gathered}
\sum_{i} s_{i}\left(L_{*}\right)=0 \\
\chi_{s}^{a b}\left(L_{*}\right)=\chi_{-s}^{c d}\left(L_{*}\right) \omega_{c}{ }^{a} \omega_{d}^{b}, \omega \in \operatorname{Aut}(g) .
\end{gathered}
$$

As a check, we note that the self K-conjugate constructions, which have $c\left(L_{*}\right)=c_{g} / 2$, are visible as $0=0$ in the general expression

$$
\sum_{i} s_{i}\left(L_{*}\right)=-\frac{\operatorname{dim}(\operatorname{dim} g+1)}{c_{g}}\left[c\left(L_{*}\right)-\frac{c_{g}}{2}\right]
$$

which is obtained from eq. (3.10) and (4.7) for the trace of $\mathcal{S}$ at a fixed point on simple $g$.

7. Connection with the T-states at level 2. The dimension of the eigenspace of $\mathcal{S}$ is $\operatorname{dim} g(\operatorname{dim} g+1) / 2$, and $\operatorname{dim} g-\operatorname{dim} h$ of these eigenvectors have been understood above as the directions of $A u t\left(L_{*}\right)$. Assuming unitarity, it is shown in Appendix $\mathrm{C}$ that the remaining eigenvalues and eigenvectors of $\mathcal{S}$ are associated to the T-states $\chi^{a b} T_{a b}^{(-2)}|0\rangle$ at level 2 ,

$$
L^{(0)} \chi_{I}^{a b} T_{a b}^{(-2)}|0\rangle=\Delta_{I} \chi_{I}^{a b} T_{a b}^{(-2)}|0\rangle
$$

where $I=1,2, \cdots, \operatorname{dim} h+\operatorname{dim} g(\operatorname{dim} g-1) / 2$, and

$$
s_{I}=1-\Delta_{I}
$$


relates the eigenvalues of $\mathcal{S}$ to the conformal weights of the T-states. For example, note that the T-states $L^{(-2)}|0\rangle, \tilde{L}^{(-2)}|0\rangle$ with $\chi^{a b}=L^{a b}$ or $\tilde{L}^{a b}$ and $\Delta=2$ or 0 correspond to the universal eigenvalues -1 or +1 discussed above. It is also shown in Appendix $\mathrm{C}$ that all the T-states are $L$-Virasoro primary except the state $L^{(-2)}|0\rangle$.

8. Quadratic deformations and $(1,0)$ states. Let $L^{a b}(\{\lambda\}) T_{a b}$ be a quadratic deformation with continuous parameters $\{\lambda\}$. It follows from the discussion above that the quadratic conformal deformation operators $\left\{\frac{\partial}{\partial \lambda_{i}} L^{a b}(\{\lambda\}) T_{a b}\right\}$ are the T-operators $\left\{\chi_{0}^{a b} T_{a b}\right\}$ with $s=0$ and hence $\Delta=1$. Since all the T-states $\chi_{0}^{a b} T_{a b}^{(-2)}|0\rangle$ with $\Delta=1$ are $L$-Virasoro primaries, it also follows that

Theorem: All quadratic deformation operators create $(1,0)$ states out of the vacuum.

This was observed for the quadratic deformations Cartan $g^{\#}, S U(2)_{4}^{\#}$ and $\left(S U(2)_{x} \times\right.$ $\left.S U(2)_{x}\right)^{\#}, x \neq 4$ in [19].

9. c-changing deformations. Since all quadratic deformation operators on the vacuum are $(1,0)$ states, it follows in particular that [19]

$$
L^{(2)} \delta L \chi_{0}^{a b} T_{a b}^{(-2)}|0\rangle=\frac{1}{4} \delta c|0\rangle=0
$$

for these states, where $\delta c$ is the change in the central charge due to an infinitesimal quadratic deformation $L_{*}^{a b}+\delta L \chi_{0}^{a b}\left(L_{*}\right)$. Then we have the following

Theorem: The master equation contains no explicitly unitary c-changing deformations.

This conclusion may be circumvented, for example, by working in the non-unitary Hilbert space of non-compact affine $g$, and projecting to unitary subspaces [32].

\section{Closed sub-flows}

The infinitesimal flow from $L$ to $L+\delta L$ during time $\delta t$ satisfies

$$
\delta L^{a b}=\beta^{a b}(L) \delta t
$$

As we will see in the following examples, iteration of the infinitesimal flow (5.1) shows that a closed sub-flow can be defined for each consistent ansatz [19] of the master equation.

a) Unitary sub-flows. The simplest consistent ansatz of the master equation is $L^{a b}=$ real in any Cartesian basis, which guarantees unitarity on $x \in N$ of affine compact $g$ [19]. The ansatz is consistent because $\beta^{a b}(L)$ is a real function of $L^{a b}$ in Cartesian coordinates. By exactly the same mechanism, the flow equation (3.11) or (5.1) maintains $L^{a b}(t)=$ real for $t>t_{0}$ when $L^{a b}\left(t_{0}\right)=$ real is the initial condition in a Cartesian frame. Put another way, the flow ansatz

$$
\dot{L}^{a b}=\beta^{a b}(L), L^{a b} \text { real in any Cartesian basis }
$$


is a closed sub-flow. This closed flow, and corresponding forms in other bases, contains all the explicitly unitary fixed points of affine-Virasoro space.

b) Flow in $S O(n)_{d i a g}$. If $L^{a b}=\delta^{a b} \lambda_{a}$ is diagonal on simple compact $g$ with $\eta_{a b}=\delta_{a b}$, then the $\beta$-function

$$
\beta^{a b}(L)=-\lambda_{a}\left(1-2 k \lambda_{a}\right) \delta^{a b}+\sum_{c d} \lambda_{c}\left(\lambda_{a}+\lambda_{b}-\lambda_{d}\right) f_{c d}^{a} f_{c d}^{b}
$$

is not diagonal in general: Such a diagonal ansatz is not generally consistent for the master equation or the flow.

In the standard Cartesian basis of $S O(n)$, there is a consistent diagonal ansatz for the master equation called $S O(n)_{\text {diag }}[24]$. The ansatz is consistent because in this basis $f_{a b}{ }^{c} \neq 0$ for at most one $c$, given $a$ and $b$, so that $\beta^{a b}(L)$ in (5.2) is also diagonal. In this case, the flow (3.11) or (5.1) remains diagonal when the initial condition is diagonal, and the flow ansatz

$$
S O(n)_{\operatorname{diag}}: \quad \dot{\lambda}_{a}=-\left[\lambda_{a}\left(1-2 k \lambda_{a}\right)-\sum_{c d} \lambda_{c}\left(2 \lambda_{a}-\lambda_{d}\right)\left(f_{c d}^{a}\right)^{2}\right]
$$

is a closed sub-flow. As reviewed below, the fixed points of this flow are isomorphic to all the graphs of graph theory [24].

c) $H$-invariant sub-flows. In this way, all known consistent ansätze can be promoted to closed sub-flows, including the basic ansatz [19, 23], the maximal symmetric sub-ansatz $[19,21]$ and the H-invariant ansätze $L_{*}^{a b}=L_{*}^{c d}\left(\omega_{H}^{-1}\right)_{c}{ }^{a}\left(\omega_{H}^{-1}\right)_{c}{ }^{b}$ of [33], for all $\omega_{H} \in H$ where $H$ is any subgroup of $G$ (not necessarily a Lie subgroup). The corresponding closed $H$-invariant sub-flow

$$
\dot{L}^{a b}=\beta^{a b}(L), L^{a b}=L^{c d}\left(\omega_{H}^{-1}\right)_{c}{ }^{a}\left(\omega_{H}^{-1}\right)_{c}^{b} \quad, \quad \forall \omega_{H} \in H \subset G
$$

follows as in [33], because $\beta(L)$ transforms as $L$ transforms under $A u t(g)$ (see eqs. (2.10) and (2.11)).

\section{Flow on the space of graphs}

As an example, we study the closed sub-flow (5.3) in $S O(n)_{\text {diag }}$, whose fixed points (solutions of the master equation) are isomorphic to the set of all graphs of order $n$ [24].

In the standard Cartesian basis of $S O(n)$, the ansatz $S O(n)_{\text {diag }}$ is [24]

$$
L^{a b}=L^{i j, k l}=\frac{l_{i j}}{2 k} \delta_{i k} \delta^{j l}
$$

where $1 \leq i<j \leq n$. In this notation ${ }^{\dagger \dagger}$, the flow equation (5.3) becomes

$$
S O(n)_{\text {diag }}: \quad i_{i j}=-l_{i j}\left(1-l_{i j}\right)+\frac{\tau}{x} \sum_{m \neq i, j}\left(l_{i m} l_{m j}-l_{i j}\left(l_{i m}+l_{j m}\right)\right), i<j
$$

${ }^{\dagger \dagger}$ The variable $L_{i j}=l_{i j} / x$ is employed in [24]. 
where the symmetry convention $l_{i j}=l_{j i}$ and $l_{i i}=0$ has been adopted to simplify the summation in (6.2). The constant $\tau$ is 1 for flow on $S O(n \geq 4)$ and 2 for $S O(3)$, and $x=2 k / \psi^{2}$ is the invariant level of $S O(n)$.

The fixed points $l_{i j}^{*}$ of $S O(n)_{d i a g}$ have been studied to all orders in $1 / x$ (semi-classical expansion) and a number of exact solutions have been obtained [24]. More generally, we collect some features of $S O(n)_{\text {diag }}$ which will be relevant in the analysis below [24]:

a) The set of fixed points in $S O(n)_{\text {diag }}$ is isomorphic to the set of all labelled graphs of order $n$, because the fixed points are characterized by their classical limit

$$
l_{i j}^{*}\left(G_{n}\right) \underset{x \rightarrow \infty}{\longrightarrow} l_{i j}^{(0)^{*}}\left(G_{n}\right)=\theta_{i j}\left(G_{n}\right), i, j=1, \cdots, n
$$

where $\theta_{i j}\left(G_{n}\right)$ is the adjacency matrix of each labelled graph $G_{n}$ of order $n$. The central charge at high level is

$$
c\left(l_{i j}^{(0)^{*}}\left(G_{n}\right)\right)=\operatorname{dim} E\left(G_{n}\right)
$$

where $\operatorname{dim} E\left(G_{n}\right)$ is the number of edges of the graph $G_{n}$. In this way, all levels of a given high- $k$ smooth conformal construction are named by a single graph.

b) The labellings of a labeled graph $G_{n}$ are physically equivalent members of $\operatorname{Aut}\left(l^{*}\left(G_{n}\right)\right)$ (the residual $S O(n)$ automorphisms of $S O(n)_{\text {diag }}$ ), so that each unlabeled graph is a physically distinct level-family of chiral conformal field theories.

c) The graph of the affine-Sugawara construction on $S O(n)$ is the complete graph (all edges) of order $n$. K-conjugation operates on the graphs as $\theta_{i j}\left(\tilde{G}_{n}\right)=1-\theta_{i j}\left(G_{n}\right)$, where $\tilde{G}_{n}$, the graph of the K-conjugate theory, is called the complement of $G_{n}$ in graph theory. The self K-conjugate constructions in (4.20) correspond to the self-complementary graphs, which satisfy $\tilde{G}_{n}=G_{n}$ as unlabeled graphs. These occur only on $S O(4 n)$ and $S O(4 n+1)$ with half-Sugawara central charge.

d) The $1 / x$ expansion and the exact solutions provide strong evidence that each fixed point $l_{i j}^{*}\left(G_{n}\right)$ is unitary (real) down to some finite critical level $x_{0}\left(G_{n}\right)$, which is generically quite low.

The C-function and flow equation (6.2) in $S O(n)_{\text {diag }}$ can also be analysed to all orders in $1 / x$, but we restrict ourselves here to the leading order

$$
\begin{gathered}
A\left(l^{(0)}\right)=\sum_{i<j}\left[3\left(l_{i j}^{(0)}\right)^{2}-2\left(l_{i j}^{(0)}\right)^{3}\right] \\
l_{i j}^{(0)}=-l_{i j}^{(0)}\left(1-l_{i j}^{(0)}\right)
\end{gathered}
$$

whose flow

$$
l_{i j}^{(0)}(t)=\frac{l_{i j}^{(0)}\left(t_{0}\right)}{l_{i j}^{(0)}\left(t_{0}\right)+\left(1-l_{i j}^{(0)}\left(t_{0}\right)\right) e^{t-t_{0}}}, t \geq t_{0}
$$


is immediately obtained for initial condition $l_{i j}^{(0)}\left(t_{0}\right)$. The schematic flow of each $l_{i j}^{(0)}$, with a minimum at 0 and a maximum at 1 , is shown in Fig. 1 . The flow pattern can also be deduced from the reduced stability matrix

$$
\mathcal{S}_{i j, k l}^{(0)}\left(l^{(0) *}\left(G_{n}\right)\right)=\delta_{i k} \delta_{j l}\left(1-2 \theta_{i j}\left(G_{n}\right)\right)
$$

which follows from the infinitesimal flow $\delta l_{i j}^{(0)}=-\mathcal{S}_{i j, k l}\left(l^{(0) *}\left(G_{n}\right)\right) \delta l_{k l}^{(0)}$. The eigenvalues of $\mathcal{S}$ are $s=-1$ for $\theta_{i j}=1$ and $s=+1$ for $\theta_{i j}=0$.

At high level, we conclude that each edge $i j\left(\theta_{i j}\left(G_{n}\right)=1\right)$ of any graph $G_{n}$ corresponds to a relevant operator $T_{a a}=T_{i j, i j}$ of the fixed point $l^{(0) *}\left(G_{n}\right)$ and each missing edge $I J$ $\left(\theta_{I J}\left(G_{n}\right)=0\right)$ of $G_{n}$ is an irrelevant operator $T_{I J, I J}$. It follows that

$$
\begin{gathered}
N_{r}\left(G_{n}\right) \equiv \text { number of relevant operators of } l^{(0) *}\left(G_{n}\right)=\operatorname{dim} E\left(G_{n}\right) \\
N_{i}\left(G_{n}\right) \equiv \text { number of irrelevant operators of } l^{(0) *}\left(G_{n}\right)=\frac{n(n-1)}{2}-\operatorname{dim} E\left(G_{n}\right) \\
N_{0}\left(G_{n}\right) \equiv \text { number of marginal operators of } l^{(0) *}\left(G_{n}\right)=0 .
\end{gathered}
$$

The K-conjugation theorems in Section 4 are clearly illustrated by the graphs. As in eqs.(4.11-13), the K-conjugate graphs $G_{n}$ and $\tilde{G}_{n}$ have relevant and irrelevant operators interchanged, since their edge sets are complementary. The graphs of self K-conjugate constructions (the self-complementary graphs) have $\operatorname{dim} E=\frac{1}{2} \operatorname{dim} g=n(n-1) / 4$, and so $N_{r}=N_{i}$. In accord with eq.(4.20a), the trace of $\mathcal{S}$ vanishes for these constructions since $|s|=1$. Moreover, each relevant operator of a self-K-conjugate graph is mapped to an irrelevant operator by the graph relabelling $\omega$, which is the $S O(n)$ automorphism in eq.(4.20).

The physical flow of graphs (excluding flow to large $l_{i j}^{(0)}$ ) is a decay of each edge of any $G_{n}$ towards a graph $G_{n}^{\prime}$ with one edge less. As simple examples, the flow of graphs in $S O(3)_{\text {diag }}$ and $S O(4)_{\text {diag }}$ is given in Figures 2 and 3, though, for simplicity, we have shown only the physically distinct, unlabeled graphs in the latter case (see also Section $8)$.

In each case, the flow of graphs is shown as beginning near the affine-Sugawara constructions, but the flow can be followed from any graph. The C-theorem (3.14) is clearly visible in the flow since $c\left(l^{(0) *}(G)\right)=\operatorname{dim} E(G)$ decays in parallel with the edges. We also remark that the graph $S O(4)^{\#}[d, 4]$ in Fig. 4 is a self K-conjugate construction. 


\section{Morse polynomials and Graph Theory}

We define the Morse polynomial $M$ and the Witten index $\nu$ for the flow on level $x$ of affine $g$ as*

$$
\begin{gathered}
M\left(t ; g_{x}\right) \equiv \sum_{F P\left(g_{x}\right)} t^{N_{r}} \\
\nu\left(g_{x}\right) \equiv M\left(-1 ; g_{x}\right)=\sum_{F P\left(g_{x}\right)}(-1)^{N_{r}}
\end{gathered}
$$

where the sums are over all the fixed points of the flow, that is, all the solutions of the master equation on level $x$ of affine $g$, and $N_{r}$ is the number of relevant operators at each fixed point. The quantities (7.1) can also be defined on any closed sub-flow.

As an example, we consider the flow of graphs in $S O(n)_{\text {diag }}$, and, following the usual strategy, we compute the quantities (7.1) in the semi-classical limit, which is high level. The results

$$
\begin{gathered}
M\left(t ; S O(n)_{\text {diag }}\right)=(1+t)^{n(n-1) / 2} \\
\nu\left(S O(n)_{\text {diag }}\right)=0
\end{gathered}
$$

follow immediately since $N_{r}=\operatorname{dim} E$ for the graphs. The form of the Morse polynomial in (7.2a) is known in graph theory as the generating function for labelled graphs. The value $\nu=0$ of the corresponding Witten index in (7.2b) is characteristic of the unbounded (cubic) C-function (6.5) since $\exp ( \pm A(L) / 12)$ cannot be a supersymmetric ground state in this case and supersymmetry must break [36].

The unbounded C-function (3.2) leads us to conjecture that $\nu\left(g_{x}\right)=0$ in general. It is also likely that $M$ and $\nu$ are invariant under smooth deformations of the level $x$, so the semiclassical results (7.2) may be exact at finite level.

We also remark on the Morse polynomials and Witten index for unlabeled graphs. These polynomials are relevant for a space which is modded by the residual $S O(n)$ automorphisms of $S O(n)_{\text {diag }}$, which keeps only one physically distinct representative of each conformal field theory. In this case, the Morse polynomials are the generating functions of unlabeled (U) graphs, e.g. [26]

$$
\begin{gathered}
M_{U}\left(t ; S O(1)_{\text {diag }}\right)=1 \\
M_{U}\left(t ; S O(2)_{\text {diag }}\right)=1+t \\
M_{U}\left(t ; S O(3)_{\text {diag }}\right)=1+t+t^{2}+t^{3} \\
M_{U}\left(t ; S O(4)_{\text {diag }}\right)=1+t+2 t^{2}+3 t^{3}+2 t^{4}+t^{5}+t^{6} \\
M_{U}\left(t ; S O(5)_{\text {diag }}\right)=1+t+2 t^{2}+4 t^{3}+6 t^{4}+6 t^{5}+4 t^{7}+2 t^{8}+t^{9}+t^{10}
\end{gathered}
$$

* Morse theory has been used to study the renormalization group flow in [34, 35]. 
where the symmetry about the center of each polynomial is K-conjugation covariance. It also follows from known results in graph theory that

$$
\nu_{U}\left(S O(n)_{\text {diag }}\right)=M_{U}\left(-1 ; S O(n)_{\text {diag }}\right)=\bar{g}_{n}
$$

where $\bar{g}_{n}$ is the number of self K-conjugate constructions (self-complementary graphs) in $S O(n)_{\text {diag }}$.

\section{Prediction of sporadic deformations}

The physical interpretation of a flow from the neighborhood of a fixed point $L_{1}^{*}$ to another fixed point $L_{2}^{*}$ is that a flow-path exists between the two conformal constructions, through the hills and valleys of the C-function. When two such constructions are flow-connected in this way, the $\mathrm{C}$-theorem guarantees that $c\left(L_{2}^{*}\right) \leq c\left(L_{1}^{*}\right)$.

At high level, the flow connections are relatively simple. For example, all unitary high-level central charges on simple compact $g$ are integer-valued from 0 to $\operatorname{dim} g$ [23], so that the selection rule $-\Delta c\left(L_{*}\right) \in N$ will be observed, as seen in the flow of graphs in Figs. 2 and 3.

As the level is lowered, however, the central charges of two or more high-level flowconnected constructions may cross, indicating that at least one local maximum and minimum have coalesced into points of inflection, which correspond to new marginal operators $^{\dagger}$ that are conformal deformations: A one-dimensional version of such a crossing is shown in Fig. 4, where the first picture is the effective C-function (6.5) of a single $l_{i j}^{(0)}$ in high-level $S O(n)_{\text {diag. }}$.

We are led to expect sporadic quadratic deformations for those levels at which two or more constructions, flow-connected at high level, have coincident central charge. The flow-connections and central charge crossings may be studied in closed sub-flows for simplicity. To be more precise, we consider only the first crossing ${ }^{\ddagger}$ as the level is lowered, since the situation is more complicated for second or higher crossings. Central charge crossings of constructions which are not high-level flow-connected should not produce deformations, since they live in path-disconnected neighborhoods of affine-Virasoro space. Moreover, the resulting conformal deformation should explicitly contain all the theories, including automorphic copies, which have coalesced at that point.

As a simple example, consider Fig. 2, which shows the flow of labelled graphs in high-level $S O(3)_{d i a g}$. Since any theory can flow to a representative of a lower theory in

\footnotetext{
${ }^{\dagger}$ The new marginal operators are distinguished from those of $A u t\left(L_{*}\right)$, which are present at all levels (or not present, with appropriate gauge fixing or ansätze).

${ }^{\ddagger}$ The first crossing of two flow-connected constructions may also mark the radius of convergence $x_{0}\left(L_{*}\right)[24]$ of the high-level expansion of these constructions.
} 
this case, any central charge crossing should be a quadratic deformation. Fig. 4 shows the central charges of these constructions at all levels, including the crossing

$$
c\left(S O(2)_{4}\right)=c\left(S O(3)_{4} / S O(2)_{x}\right)=1
$$

at level four. In fact, this is the known quadratic deformation $S U(2)_{4}^{\#}[17]$.

According to the graph flow in Fig. 2, all six graphs (3 automorphically equivalent copies of $S O(2)$ and 3 copies of $S O(3) / S O(2))$ are coalescing at level 4, so all six theories must be points of $S U(2)_{4}^{\#}$. To check this, we used an alternate form ${ }^{\S}$ of $S U(2)_{4}^{\#}$

$$
\begin{gathered}
T\left(L^{\#}(\phi)\right)=\frac{1}{4 \alpha^{2}} \sum_{i<j}^{3} l_{i j}(\phi)_{*}^{*} J_{i j}^{2}{ }_{*}^{*}, c=1 \\
l_{12}(\phi)=\frac{1}{3}(1-\sqrt{3} \cos \phi+\sin \phi) \\
l_{13}(\phi)=\frac{1}{3}(1-2 \sin \phi) \\
l_{23}(\phi)=\frac{1}{3}(1+\sqrt{3} \cos \phi+\sin \phi)
\end{gathered}
$$

with $\alpha^{2}=\psi^{2}$ and $0 \leq \phi<2 \pi$, which shows that this manifold of fixed points is a circle $~$. The circle is shown in Fig. 5, with the location of all six rational constructions predicted by the method. In connection with our remarks in Sections 3 and 4, we have also checked that the symmetry group $O(2)$ of the circle is an accidental symmetry of the $S O(3)$ C-function at level 4.

Moving on, we apply the method to $S O(4)_{\text {diag. }}$. The central charges of all the level families in $S O(4)_{\text {diag }}$ are shown in Fig. 6, including the self K-conjugate construction $S O(4)^{\#}[d, 4]$. As the level $x$ is lowered from asymptotic values, the first central charge crossings

$$
S O(4) /(S O(2) \times S O(2)) \sim S O(3), S O(4) / S O(3) \sim S O(2) \times S O(2)
$$

are observed at level $x=3+\sqrt{13}$. It is clear from the graph flow of Fig. 4 that these pairs of constructions are not flow-connected at high level, so no conformal deformations are predicted at these points. To test this negative prediction, we checked that the stability matrices in $S O(4)_{d i a g}$ of these constructions have no zero eigenvalues at this level, as expected.

\footnotetext{
$\S_{\text {The connection with the form of } S U}(2)_{4}^{\#}$ in [19] is: $l_{12}=4 \alpha^{4} \lambda, l_{13}=4 \alpha^{2}\left(L^{\alpha,-\alpha}-L^{\alpha, \alpha}\right), l_{23}=$ $4 \alpha^{2}\left(L^{\alpha,-\alpha}+L^{\alpha, \alpha}\right),-1 / 12 \leq \lambda \alpha^{4} \leq 1 / 4$.

"The action of K-conjugation on the circle of Fig. 5 is reflection through the origin. The action of the residual automorphisms $\left(S_{3}\right)$ of $S O(3)_{\text {diag }}$ is reflection about each of the three axes shown in the Figure. It follows that the points marked by crosses in Fig. 5 are automorphically equivalent copies of a single self K-conjugate construction in $S U(2)_{4}^{\#}$. Modding by $S_{3}$, each $\pi / 3$ arc from a $g / h$ to an $h^{\prime}$ is a fundamental region.
} 
On the other hand, the triple crossing at level 4

$$
x=4: S O(4) /(S O(2) \times S O(2)) \sim S O(4)^{\#}[d, 4] \sim S O(2) \times S O(2)
$$

involves constructions which, according to oval (a) of Fig. 4, are flow-connected at high level. We predict, therefore, a new quadratic deformation, which we call

$$
S O(4)_{4}^{\#} \text { with } c=2 .
$$

As a check on the prediction, we computed the stability matrices in $S O(4)_{\text {diag }}$ of the constructions in (8.3). They all show zero eigenvalues at level four as expected. To learn more about $S O(4)_{4}^{\#}$, we consider in Fig. 6a the flow of labelled graphs within oval (a) of Fig. 4. The predicted deformation should contain all 18 constructions as special points, including the 12 copies of the self K-conjugate construction. Moreover, we can predict that $S O(4)_{4}^{\#}$ will be closed under K-conjugation, as was $S U(2)_{4}^{\#}$, since the set of graphs in Fig. 6a is closed under K-conjugation.

There are two more first crossings at level 2,

$$
\begin{gathered}
x=2, c=2: S O(4) / S O(2) \sim S O(4) / S O(3) / S O(2) \sim S O(3) \\
x=2, c=1: S O(2) \sim S O(3) / S O(2) \sim S O(4) / S O(3)
\end{gathered}
$$

which, according to ovals (b) and (c) of Fig. 4, are flow-connected at high level. Since the constructions in (8.5a) are related to those in $(8.5 \mathrm{~b})$ by K-conjugation, we predict the K-conjugate pair of quadratic deformations

$$
S O(4)_{2}^{\#} \text { with } c=1, S O(4) / S O(4)_{2}^{\#} \text { with } c=2 .
$$

Further structure in $S O(4)_{2}^{\#}$ is seen in Fig. 6b, which shows the labelled graph flow in oval (b) of Fig. 4. All 22 constructions should be found as points in $S O(4)_{2}^{\#}$. It is possible that the known deformation $S U(2)_{4}^{\#}$ is irregularly embedded in $S O(4)_{2}^{\#}$, but the predicted deformation $S O(4)_{2}^{\#}$, which contains $S O(4) / S O(3)$, is clearly larger than $S U(2)_{4}^{\#}$. The predicted deformation may also be related to the known deformation $\left(S U(2)_{x} \times S U(2)_{x}\right)^{\#}$ at $x=2[19]$, but the two cannot be identical since the known deformation is closed under K-conjugation.

There are many central charge crossings in affine-Virasoro space. As an example, the list

$$
\begin{gathered}
c\left(S U(3)_{3} /(U(1) \times U(1))\right)=c(U(1) \times U(1))=2 \\
c\left(S O(2 n+1)_{2} /[S O(2)]^{n}\right)=c\left([S O(2)]^{n}\right)=n \\
c\left(S O(2 n)_{2} /[S O(2)]^{n}\right)=c\left([S O(2)]^{n-1}\right)=n-1 \\
c\left([S O(2)]^{n}\right)=c\left(S O(2 n)_{2} /[S O(2)]^{n-1}\right)=n
\end{gathered}
$$


records the crossings of simple $g_{x} /$ Cartan $g$ with $U(1)^{p}, 0<p \leq \operatorname{rank} g, x \in N$. This suggests the candidate deformations

$$
\begin{gathered}
S U(3)_{3}^{\#}, c=2 ; S O(2 n+1)_{2}^{\#}, c=n \\
S O(2 n)_{2}^{\#}, c=n-1 ; S O(2 n) / S O(2 n)_{2}^{\#}, c=n
\end{gathered}
$$

but it is generally difficult to verify high-level flow connection and first crossing, even in closed sub-flows, unless all the fixed points of the subspace are known. The list (8.8) is at least partially correct, however, since the known [23] deformation $S O(2 n+1)_{2}^{\#}[d, 6]$ is presumably included in $S O(2 n+1)_{2}^{\#}$. Moreover, we will report elsewhere [30] a sub-flow in which $S U(3) /[U(1)]^{2}$ and $[U(1)]^{2}$ are flow-connected with a first crossing at level three, and we will obtain the exact form of the predicted deformation $S U(3)_{3}^{\#}$.

We finally remark that all known sporadic quadratic deformations, including the predictions of this section, are found on integer level of affine $g$ such that the affineSugawara construction has integer central charge.

\section{Conclusions}

We have constructed an exact C-function on affine-Virasoro space, and the associated flow on the space of theories satisfies a C-theorem. The fixed points of the flow are the conformal field theories of the Virasoro master equation. The flow defines relevant, irrelevant and marginal operators at the fixed points, and emphasizes the relations (and collisions) among the conformal field theories of affine-Virasoro space. As an example, the system was solved for high level $S O(n)_{\text {diag }}$, which is a flow on the space of graphs.

Although the resemblance is striking, it is not yet clear whether the $L^{a b}$ flow on affine-Virasoro space is an exact renormalization group equation. The $L^{a b}$ flow does not correspond to covariant perturbation of the WZW theory by the marginal operators $g^{a b} J_{a} \bar{J}_{b}$, since all $L^{a b}$ flow directions are relevant near the affine-Sugawara constructions, and the eigenvalues of the stability matrix are reparametrization invariants. Since $L_{*}^{a b} J_{a} J_{b}$ is a set of chiral stress tensors, it is plausible that the $L^{a b}$ flow is the renormalization group flow computed in a light-cone Hamiltonian perturbation theory.

In this connection, the quantum analogue of our system is constructed as follows. The natural Langevin equation $\|$

$$
\dot{L}_{\eta}^{a b}=\beta^{a b}\left(L_{\eta}\right)+P^{a b, c d} \eta_{c d}, \quad\left\langle\eta_{a b}(t) \eta_{c d}\left(t^{\prime}\right)\right\rangle=P_{a b, c d} \delta\left(t-t^{\prime}\right)
$$

\footnotetext{
"Langevin quantization of the renormalization group equation was suggested in [37] and studied in [35]. The normalization of the noise in (9.1) follows the convention of [38].
} 
does not equilibrate to its formal Boltzmann factor $\exp (-A(L) / 6)$ because the C-function $A(L)$ is bottomless. The Greensite-Halpern-Marinari-Parisi stabilization [39] substitutes the Fokker-Plank Hamiltonian and/or its equivalent fifth-time action

$$
\begin{gathered}
H_{5} \equiv \frac{1}{2} P^{c d, a b} Q_{c d}^{\dagger} Q_{a b}, Q_{a b} \equiv \frac{1}{i} \frac{\partial}{\partial L^{a b}}+i P_{a b, c d} \beta^{c d} \\
S_{5} \equiv \frac{1}{2} \int d t\left[\dot{L}^{a b} P_{a b, c d} \dot{L}^{c d}+\beta^{a b} P_{a b, c d} \beta^{c d}+\frac{\partial}{\partial L^{a b}} \beta^{a b}\right] .
\end{gathered}
$$

This stabilized system chooses the normalizable positive energy-ground state of $H_{5}$ and, with the usual fermionic scaffolding, corresponds to broken supersymmetry. Moreover, for fixed initial and final conditions, the averages of the fifth-time action (9.2b) may be computed from its non-equilibrating Nicolai map (9.1) [38, 40]: With $L\left(t_{1}\right)=L_{\eta}\left(t_{1}\right)=L_{1}$ and $L\left(t_{2}\right)=L_{2}$, the relation [38]

$$
\left\langle\exp \int_{t_{1}}^{t_{2}} \mathrm{~d} t J_{a b} L^{a b}\right\rangle_{S_{5}}=\left\langle\delta\left(L_{2}-L_{\eta}\left(t_{2}\right)\right) \exp \int_{t_{1}}^{t_{2}} \mathrm{~d} t J_{a b} L_{\eta}^{a b}\right\rangle_{\eta} /\left\langle\delta\left(L_{2}-L_{\eta}\left(t_{2}\right)\right)\right\rangle_{\eta}
$$

is completely general for equilibrating or non-equilibrating maps.

\section{Acknowledgements}

We thank O. Alvarez, P. Windey and especially J. Lepowsky for helpful conversations. 


\section{Appendix A. OPE's and associativity identities}

In this appendix, we collect operator products among some composite operators in the affine vacuum module. The basic OPE's were given in [15], but the treatment of spin 3 operators was incomplete, so we list the complete results and also collect some useful associativity identities among OPE coefficients.

The OPE of two currents is

$$
\begin{gathered}
J_{a}(z) J_{b}(w)=\frac{G_{a b}}{(z-w)^{2}}+i f_{a b}{ }^{c}\left[\frac{1}{z-w}+\frac{1}{2} \partial_{w}+\frac{1}{6}(z-w) \partial_{w}^{2}\right] J_{c}(w)+ \\
+\left[1+\frac{1}{2}(z-w) \partial_{w}\right] T_{a b}(w)+(z-w) X_{a b}(w)+\mathcal{O}\left[(z-w)^{2}\right]
\end{gathered}
$$

which defines the spin-2 composite operators $T_{a b}(z)=T_{b a}(z)$ and the spin-3 operators $X_{a b}(z)=-X_{b a}(z)$, both operators being quasiprimary. The 2-point functions of $T_{a b}$ and $X_{a b}$

$$
\left\langle T_{a b}(z) T_{c d}(w)\right\rangle=\frac{P_{a b, c d}}{(z-w)^{4}},\left\langle X_{a b}(z) X_{c d}(w)\right\rangle=\frac{H_{a b, c d}}{(z-w)^{6}}
$$

are computed from (A.1), where

$$
H_{a b, c d}=G_{d[a} G_{b] c}-\frac{1}{6} f_{a b}{ }^{e} f_{c d}^{f} G_{e f} \quad, \quad H_{a b, c d}=H_{c d, a b}
$$

and $P_{a b, c d}$ is given in eq. $(2.6 \mathrm{~b})$.

Next, we compute the OPE of $T_{a b}$ with $J_{c}$,

$$
\begin{gathered}
T_{a b}(z) J_{c}(w)=M_{a b, c}{ }^{d}\left[\frac{1}{(z-w)^{2}}+\frac{1}{(z-w)} \partial_{w}+\frac{1}{2} \partial_{w}^{2}\right] J_{d}(w)+ \\
N_{a b, c} d e\left[\frac{1}{(z-w)}+\frac{3}{4} \partial_{w}\right] T_{d e}(w)+W_{a b c}(w)+K_{a b, c}^{d e} X_{d e}(w)+\mathcal{O}(z-w)
\end{gathered}
$$

where

$$
\begin{gathered}
M_{a b, c}{ }^{d}=\delta_{(a}^{d} G_{b) c}+\frac{1}{2} f_{e(a}{ }^{d} f_{b) c}{ }^{e} \\
N_{a b, c}{ }^{d e}=\frac{i}{2} \delta_{\left(a^{d} f_{b) c}{ }^{e)}\right.}
\end{gathered}
$$

and (A.4) serves as a definition of the spin-3 composite operators $W_{a b c}(z)$.

Finally,

$$
\begin{gathered}
T_{a b}(z) T_{c d}(w)=\frac{P_{a b, c d}}{(z-w)^{4}}+Q_{a b, c d} e\left[\frac{1}{(z-w)^{3}}+\frac{1}{2(z-w)^{2}} \partial_{w}+\frac{1}{6(z-w)} \partial_{w}^{2}\right] J_{e}(w)+ \\
+R_{a b, c d} e f\left[\frac{1}{(z-w)^{2}}+\frac{1}{2(z-w)} \partial_{w}\right] T_{e f}(w)+ \\
+S_{a b, c d} \text { efg } \frac{W_{e f g}(w)}{(z-w)}+U_{a b, c d} \frac{\text { ef } \frac{X_{e f}(w)}{(z-w)}+\mathcal{O}(1)}{\text { ( } 1 \text {. }}
\end{gathered}
$$


where $Q, S$ and $U$ are antisymmetric under the interchange $(a b \leftrightarrow c d)$ while $R$ is symmetric. This OPE differs by the $X_{a b}$ term from the one given in [15], where the explicit form of $Q, R$ and $S$ is given. The correct value of $S$ is $1 / 6$ of the result given in [15]. The conclusions of references $[15,19]$ are not changed by the extra term since $U$ is antisymmetric and $X_{a b}^{m \geq-2}|0\rangle=0$.

Some relevant 3-point functions are

$$
\begin{gathered}
\left\langle J_{a}\left(z_{1}\right) J_{b}\left(z_{2}\right) J_{c}\left(z_{3}\right)\right\rangle=\frac{i f_{a b}{ }^{e} G_{e c}}{z_{12} z_{13} z_{23}} \\
\left\langle T_{a b}\left(z_{1}\right) J_{c}\left(z_{2}\right) J_{d}\left(z_{3}\right)\right\rangle=\frac{P_{a b, c d}}{z_{12}^{2} z_{13}^{2}} \\
\left\langle T_{a b}\left(z_{1}\right) T_{c d}\left(z_{2}\right) J_{e}\left(z_{3}\right)\right\rangle=\frac{Q_{a b, c d} G_{f e}}{z_{12}^{3} z_{13} z_{23}} \\
\left\langle T_{a b}\left(z_{1}\right) T_{c d}\left(z_{2}\right) T_{e f}\left(z_{3}\right)\right\rangle=\frac{R_{a b, c d}{ }^{g h} P_{g h, e f}}{z_{12}^{2} z_{13}^{2} z_{23}^{2}} .
\end{gathered}
$$

A partial list of associativity identities among the OPE coefficients is as follows:

$$
\begin{aligned}
& M_{a b, c}{ }^{e} G_{e d}=P_{a b, c d} \\
& N_{c d, e^{g h}} P_{a b, g h}=Q_{a b, c d}{ }^{g} G_{g e} \equiv Q_{a b, c d, e} \\
& K_{a b, c}{ }^{g h} H_{g h, d e}=\frac{i}{2} P_{a b, g[d} f_{e] c}{ }^{g} \\
& Q_{a b, c d, e}=i P_{a b, g(c} f_{d) e^{g}} \\
& N_{a b, f}^{g h} Q_{g h, c d, e}+N_{c d, f}^{g h} Q_{a b, g h, e}=-i f_{e f}^{g} Q_{a b, c d, g} \\
& U_{a b, c d}^{g h} H_{g h, e f}=\left[M_{a b, f^{g}} P_{c d, g e}+\frac{1}{6} N_{a b, f}^{g h} Q_{c d, g h, e}-(a b \leftrightarrow c d)\right] \\
& R_{a b, c d}^{g h} P_{g h, e f}=R_{a b, e f}^{g h} P_{g h, c d} \\
& R_{a b, c d}{ }^{g h} P_{g h, e f}=M_{a b, f}^{g} P_{c d, g e}+\frac{1}{2} N_{a b, f}^{g h} Q_{c d, g h, e}+(a b \leftrightarrow c d) .
\end{aligned}
$$

We remark in particular that the covariant tensor $R_{a b, c d, e f} \equiv R_{a b, c d}{ }^{g h} P_{g h, e f}$ is completely symmetric under the interchange of any of its indices, according to (A.18).

Some other useful identities are

$$
\begin{gathered}
R_{a b, c d}^{e f} G_{e f}=2 P_{a b, c d}, \quad L_{g}^{e f} R_{e f, a b}{ }^{c d}=2 \delta_{a b}^{c d} \\
L_{g}^{c d} P_{c d, a b}=G_{a b}, \quad L_{g}^{c d} Q_{c d, a b}{ }^{e}=0
\end{gathered}
$$

where $L_{g}^{a b}$ is the inverse inertia tensor (2.7) of the affine-Sugawara construction. 


\section{Appendix B: Covariant Structures on Affine-Virasoro Space}

Covariant forms of the C-function (3.2), the master equation (2.6) and the flow equation (3.11) under reparametrizations $L^{\prime}(L)$ are quite ordinary. The metric $P_{a b, c d}(L)$ and

the third rank tensor $R_{a b, c d}$ ef $(L)$ are obtained in an arbitrary frame $L^{\prime}(L)$ by transforming from the original frame of the text, for example

$$
P_{a b, c d}^{\prime}\left(L^{\prime}\right)=\frac{\partial L^{e f}}{\partial L^{\prime a b}} \frac{\partial L^{g h}}{\partial L^{\prime c d}} P_{e f, g h}
$$

The metric has vanishing Riemann tensor in any frame since it is globally flat in the original frame.

The action is defined to transform as a scalar

$$
A^{\prime}\left(L^{\prime}\right)=A(L)
$$

which determines its form in any frame. The covariant flow equation is

$$
\dot{L}^{a b}=-\frac{1}{12} P^{a b, c d}(L) \frac{\partial A(L)}{\partial L^{c d}} \equiv \beta^{a b}(L)
$$

since, as defined, the $\beta$-function is a contravariant vector. The covariant form of the master equation is $\beta^{a b}\left(L_{*}\right)=0$ and the central charge is invariant under reparametrizations. The covariant flow near a fixed point $L=L_{*}+\delta L$ is

$$
\begin{gathered}
D_{t} \delta L^{a b}=-\mathcal{S}^{a b}{ }_{c d}\left(L_{*}\right) \delta L^{c d} \\
D_{t} \delta L^{a b} \equiv \partial_{t} \delta L^{a b}+\delta L^{c d} \Gamma_{c d, e f}{ }^{a b} \dot{L}^{e f} \\
\mathcal{S}^{a b}{ }_{c d}(L) \equiv-D_{c d} \beta^{a b}(L)=\frac{1}{12} P^{a b, e f} D_{c d} \frac{\partial A(L)}{\partial L^{e f}}
\end{gathered}
$$

where $D_{a b}$ is the covariant derivative with respect to $L^{a b}, D_{t}$ is the covariant time derivative along a line of flow and $\Gamma_{a b, c d}$ ef is the Christoffel connection of $P_{a b, c d}(L)$. Moreover, the stability matrix $\mathcal{S}$ in (B.6) is a mixed second rank tensor, so that its eigenvalues are reparametrization invariant.

The invariant distance along a path $\mathcal{P}$ between two points $L_{1}$ and $L_{2}$ in Affine-Virasoro space is

$$
d\left(L_{1}, L_{2} ; \mathcal{P}\right) \equiv \int_{s_{1}}^{s_{2}} d s \sqrt{\dot{L}^{a b} P_{a b, c d}(L) \dot{L}^{c d}}
$$

where overdot is derivative with respect to $s$, which parametrizes the path $L(s)$ from $L_{1}$ to $L_{2}$. The distance is independent of reparametrizations $s^{\prime}(s), L^{\prime}(L)$ and is real on $x_{I} \in N$ of affine compact $g$. The "flow distance" between two points on a line of flow is obtained from (B.7) with $s=t$. 


\section{Appendix C. The stability matrix and the affine vacuum module.}

In this appendix we analyze level two of the affine vacuum module, as organized by some solution to the master equation. In particular, we relate a subset of eigenvalues of the stability matrix $\mathcal{S}$ to a subset of conformal weights at this level. Let $V_{2}$ be the vector space spanned by the states $J_{a}^{(-2)}|0\rangle$ and $T_{a b}^{(-2)}|0\rangle$ with mode number two. We restrict our discussion to integer level $x>1$ of affine compact $g$ so that all the states in $V_{2}$ are linearly independent [31] and $\operatorname{dim} V_{2}=D+D(D+1) / 2$, where $D=\operatorname{dim} g$.

Let $L_{*}^{a b}$ be a unitary solution of the master equation and $L^{(n)}=L_{*}^{a b} T_{a b}^{(n)}$ the modes of the corresponding Virasoro operator, so that $\left(L^{(n)}\right)^{\dagger}=L^{(-n)}$. Let $\tilde{L}^{(n)}$ be the commuting K-conjugate Virasoro algebra $\tilde{L}^{(n)}=L_{g}^{(n)}-L^{(n)}$. The following commutation relations are obtained from the OPE's of Appendix A,

$$
\begin{gathered}
{\left[L^{(m)}, J_{a}^{(n)}\right]=-n M_{a}^{b} J_{b}^{(m+n)}+N_{a}^{b c} T_{b c}^{(m+n)}} \\
{\left[\tilde{L}^{(m)}, J_{a}^{(n)}\right]=-n\left(\delta_{a}^{b}-M_{a}^{b}\right) J_{b}^{(m+n)}-N_{a}^{b c} T_{b c}^{(m+n)}} \\
{\left[L^{(m)}, T_{a b}^{(n)}\right]=\frac{P_{a b}}{6}\left(m^{3}-m\right) \delta_{m+n, 0}+\frac{Q_{a b}^{c}}{6}\left(m^{2}+n^{2}-m n-1\right) J_{c}^{(m+n)}+} \\
+\frac{(m-n)}{2} R_{a b}{ }^{c d} T_{c d}^{(m+n)}+S_{a b}{ }^{c d e} W_{c d e}^{(m+n)}+U_{a b}{ }^{c d} X_{c d}^{(m+n)} \\
{\left[L^{(m)}, \tilde{L}^{(n)}\right]=0}
\end{gathered}
$$

where the quantities

$$
\begin{gathered}
P_{a b} \equiv L_{*}^{c d} P_{a b, c d}, M_{a}^{b} \equiv L_{*}^{c d} M_{c d, a}{ }^{b}, P_{a b}=M_{a}{ }^{c} G_{c b} \\
N_{a}^{b c} \equiv L_{*}^{d e} N_{d e, a}^{b c}, Q_{a b}{ }^{c} \equiv L_{*}^{d e} Q_{a b, d e}{ }^{c}, R_{a b}{ }^{c d} \equiv L_{*}^{e f} R_{a b, e f}{ }^{c d}
\end{gathered}
$$

are linear in $L_{*}^{a b}$. We also need $J_{a}^{m \geq 0}|0\rangle=T_{a b}^{m \geq-1}|0\rangle=W_{a b c}^{m \geq-2}|0\rangle=X_{a b}^{m \geq-2}|0\rangle=0$, where $|0\rangle$ is the affine vacuum.

Consider first the following states in $V_{2}$,

$$
\begin{gathered}
\left|\mathcal{L}_{a}\right\rangle \equiv L^{(-1)} J_{a}^{(-1)}|0\rangle=M_{a}^{b} J_{b}^{(-2)}|0\rangle+N_{a}^{b c} T_{b c}^{(-2)}|0\rangle \\
\left|\tilde{\mathcal{L}}_{a}\right\rangle \equiv \tilde{L}^{(-1)} J_{a}^{(-1)}|0\rangle=\left(\delta_{a}^{b}-M_{a}^{b}\right) J_{b}^{(-2)}|0\rangle-N_{a}^{b c} T_{b c}^{(-2)}|0\rangle
\end{gathered}
$$

and the inverse relations

$$
\begin{gathered}
J_{a}^{(-2)}|0\rangle=\left|\mathcal{L}_{a}\right\rangle+\left|\tilde{\mathcal{L}}_{a}\right\rangle \\
N_{a}{ }^{b c} T_{b c}^{(-2)}|0\rangle=\left(\delta_{a}^{b}-M_{a}^{b}\right)\left|\mathcal{L}_{b}\right\rangle-M_{a}{ }^{b}\left|\tilde{\mathcal{L}}_{b}\right\rangle .
\end{gathered}
$$

Let $V_{\mathcal{L}} \subset V_{2}$ and $V_{\tilde{\mathcal{L}}} \subset V_{2}$ be the vector spaces spanned by $\left|\mathcal{L}_{a}\right\rangle$ and $\left|\tilde{\mathcal{L}}_{a}\right\rangle$ respectively.

Proposition 1: $V_{\mathcal{L}}$ is orthogonal to $V_{\tilde{\mathcal{L}}}$. 
Proof: $\left\langle\mathcal{L}_{a} \mid \tilde{\mathcal{L}}_{b}\right\rangle=\left\langle 0\left|J_{a}^{(1)} L^{(1)} \tilde{L}^{(-1)} J_{b}^{(-1)}\right| 0\right\rangle=\left\langle 0\left|J_{a}^{(1)} \tilde{L}^{(-1)} L^{(1)} J_{b}^{(-1)}\right| 0\right\rangle=0$, using (C.7), (C.4) and (C.1).

Let $N_{0}, N_{1}$ be the number of zero, one eigenvalues of the matrix $M_{a}^{b}$.

Proposition 2: $\operatorname{dim} V_{\mathcal{L}}=D-N_{0} \equiv \operatorname{rank}(M)$, and $\operatorname{dim} V_{\tilde{\mathcal{L}}}=D-N_{1} \equiv \operatorname{rank}(1-M)$.

Proof: Evaluate the inner products

$$
\begin{gathered}
\left\langle\mathcal{L}_{a} \mid \mathcal{L}_{b}\right\rangle=\left\langle 0\left|J_{a}^{(1)} L^{(1)} L^{(-1)} J_{b}^{(-1)}\right| 0\right\rangle=2 M_{a}{ }^{c} G_{c b} \\
\left\langle\tilde{\mathcal{L}}_{a} \mid \tilde{\mathcal{L}}_{b}\right\rangle=\left\langle 0\left|J_{a}^{(1)} \tilde{L}^{(1)} \tilde{L}^{(-1)} J_{b}^{(-1)}\right| 0\right\rangle=2\left(\delta_{a}^{c}-M_{a}^{c}\right) G_{c b} .
\end{gathered}
$$

It follows from (C.9a) that the number of linear relations among the states $\left|\mathcal{L}_{a}\right\rangle$ is equal to the number $N_{0}$ of zero eigenvalues of $M_{a}{ }^{c}$, since $G_{a b}$ is invertible. Then, $\operatorname{dim} \operatorname{span}\left\{\left|\mathcal{L}_{a}\right\rangle\right\}=$ $D-N_{0}=\operatorname{rank}(M)$. The conclusion for $\operatorname{dim} V_{\tilde{\mathcal{L}}}$ follows in the same way from (C.9b).

Next, evaluate $\left\langle\mathcal{L}_{a} \mid \tilde{\mathcal{L}}_{b}\right\rangle=0$ using the right sides of (C.7a,b) to obtain

$$
N_{a}^{c d} N_{b}^{e f} P_{c d, e f}=2 M_{a}^{d} G_{d e}\left(M_{b}^{c}-\delta_{b}^{c}\right) .
$$

Consider $\left\{N_{a}^{b c}\right\}$ as a collection of $D$ vectors (indexed by a), each with $D(D+1) / 2$ components (indexed by $b c$ ). The inner product on these vectors,

$$
\left\langle N_{a} \mid N_{b}\right\rangle \equiv\left(N_{a}^{c d}\right)^{*} P_{c d, e f} N_{b}^{e f}=-N_{a}^{c d} P_{c d, e f} N_{b}^{e f}
$$

is positive because $P_{a b, c d}$ is positive definite and $N_{a}^{c d}$ is pure imaginary (see (A.6)). This is the inner product in the space of right eigenvectors of the stability matrix (see eq.(4.5a)).

Proposition 3: $\left\{N_{a}^{b c}\right\}$ generates a vector space $V_{N}$ with $\operatorname{dim} V_{N}=D-N_{0}-N_{1}$.

Proof: It follows from (C.10) that the matrix of inner products $\left\langle N_{a} \mid N_{b}\right\rangle$ has $N_{0}+N_{1}$ zero eigenvalues, which correspond to the zero, one eigenvalues of $M$.

Remark: In [33], it is shown that $N_{0}+N_{1}=\operatorname{dim} h$ where $h \subset g$ is the Lie subgroup symmetry of the construction $L_{*}^{a b}$.

The stability matrix

$$
\mathcal{S}^{a b}{ }_{c d}=\left[\frac{1}{2}\left(\delta^{a}{ }_{c} \delta^{b}{ }_{d}+\delta^{a}{ }_{d} \delta^{b}{ }_{c}\right)-R_{c d}{ }^{a b}\right]
$$

is defined in (4.1).

Proposition 4: $\mathcal{S}^{a b}{ }_{c d}$ has at least $\operatorname{dim} V_{N}$ zero eigenvalues. The right eigenspace corresponding to these eigenvalues is $V_{N}$.

Proof: We compute $L^{(0)} N_{a}^{b c} T_{b c}^{(-2)}|0\rangle$ in two ways. The first computation uses (C.8b), (C.1) and (C.7),

$$
L^{(0)} N_{a}^{b c} T_{b c}^{(-2)}|0\rangle=\left(\delta_{a}^{b}-M_{a}^{b}\right) L^{(0)}\left|\mathcal{L}_{b}\right\rangle-M_{a}^{b}\left|\tilde{\mathcal{L}}_{b}\right\rangle=
$$




$$
=\left(M_{a}^{b}-\left(M^{2}\right)_{a}{ }^{b}\right) J_{b}^{(-2)}|0\rangle+N_{a}^{b c} T_{b c}^{(-2)}|0\rangle .
$$

The second computation uses (C.3),

$$
L^{(0)} N_{a}^{b c} T_{b c}^{(-2)}|0\rangle=N_{a}^{b c}\left[\frac{1}{2} Q_{b c}{ }^{d} J_{d}^{(-2)}|0\rangle+R_{b c}{ }^{d e} T_{d e}^{(-2)}|0\rangle\right] .
$$

Matching the right hand sides of (C.13a,b) we obtain (C.10) and

$$
N_{a}^{b c}=N_{a}^{d e} R_{d e}^{b c}
$$

It follows from (C.12) and (C.14) that $V_{N}$ is a right eigenspace of $\mathcal{S}$ with zero eigenvalue.

Proposition 5: When $\psi^{a}$ is a left eigenvector of $M_{a}{ }^{b}$ with an eigenvalue which is either one or zero, then $\psi^{a} N_{a}^{b c}=0$.

Proof: Consider first the case when $\psi^{a} M_{a}^{b}=\psi^{b}$. Compute the norm of the state $\psi^{a} \tilde{L}^{(-1)} J_{a}^{(-1)}|0\rangle$,

$$
\begin{gathered}
\|\left.\psi^{a} \tilde{L}^{(-1)} J_{a}^{(-1)}|0\rangle\right|^{2}=\psi^{a} \psi^{b}\left\langle 0\left|J_{a}^{(1)} \tilde{L}^{(1)} \tilde{L}^{(-1)} J_{b}^{(-1)}\right| 0\right\rangle= \\
=2 \psi^{a} \psi^{b}\left(\delta_{a}^{c}-M_{a}^{c}\right)\left\langle 0\left|J_{a}^{(1)} J_{c}^{(-1)}\right| 0\right\rangle=0
\end{gathered}
$$

which implies that the state is identically zero, $\tilde{L}^{(-1)} \psi^{a} J_{a}^{(-1)}|0\rangle=0$. On the other hand, this state has the explicit form

$$
0=\tilde{L}^{(-1)} \psi^{a} J_{a}^{(-1)}|0\rangle=\psi^{a}\left[\left(\delta_{a}^{b}-M_{a}^{b}\right) J_{b}^{(-2)}-N_{a}^{b c} T_{b c}^{(-2)}\right]|0\rangle=-\psi^{a} N_{a}^{b c} T_{b c}^{(-2)}|0\rangle
$$

which implies that $\psi^{a} N_{a}^{b c}=0$. The same follows for the case $\psi^{a} M_{a}^{b}=0$ using the state $L^{(-1)} \psi^{a} J_{a}^{(-1)}|0\rangle=0$.

Corollary 1: Let $\psi_{1}^{a}\left(\psi_{0}^{a}\right)$ be an eigenvector of $M_{a}^{b}$ with eigenvalue one (zero). Then

$$
\begin{gathered}
{\left[L^{m}, \psi_{1}^{a} J_{a}^{(n)}\right]=-n \psi_{1}^{a} J_{a}^{(m+n)},\left[\tilde{L}^{(m)}, \psi_{1}^{a} J_{a}^{(n)}\right]=0} \\
{\left[L^{(m)}, \psi_{0}^{a} J_{a}^{(n)}\right]=0,\left[\tilde{L}^{(m)}, \psi_{0}^{a} J_{a}^{(n)}\right]=-n \psi_{0}^{a} J_{a}^{(m+n)}}
\end{gathered}
$$

follows from Proposition 5: The $L_{*}$ theory contains a $(1,0)$ operator (current) for each unit eigenvalue of $M_{a}^{b}\left(L_{*}\right)$, and the current commutes with the K-conjugate theory $\tilde{L}_{*}$. The conclusions for $L_{*}$ and $\tilde{L}_{*}$ are reversed for zero eigenvalues of $M_{a}^{b}\left(L_{*}\right)$.

Consider next the orthogonal decomposition $V_{2}=V_{\mathcal{L}} \oplus V_{\tilde{\mathcal{L}}} \oplus V_{T}$, which defines the subspace $V_{T}$ of all vectors in $V_{2}$ orthogonal to $V_{\mathcal{L}}$ and $V_{\tilde{\mathcal{L}}}$. The dimension of $V_{T}$ is $\operatorname{dim} V_{T}=\operatorname{dim} V_{2}-\operatorname{dim} V_{\mathcal{L}}-\operatorname{dim} V_{\tilde{\mathcal{L}}}=N_{0}+N_{1}-D+D(D+1) / 2$. We now give a characterization of the vectors in $V_{T}$.

Proposition 6: Iff a vector is in $V_{T}$ then the vector has the form $\chi^{a b} T_{a b}^{(-2)}|0\rangle$ where $N_{e} \overline{{ }^{a} \chi^{c d} P_{a b, c d}=0}, \forall e$. 
Proof: The general vector in $V_{T}$ is $|T\rangle \equiv \chi^{a b} T_{a b}^{(-2)}|0\rangle+B^{a} J_{a}^{(-2)}|0\rangle$. We require that $|T\rangle$ is orthogonal to $V_{\mathcal{L}} \oplus V_{\tilde{\mathcal{L}}}$,

$$
\begin{gathered}
\left\langle\mathcal{L}_{a} \mid T\right\rangle=2 P_{a b} B^{b}-N_{a}^{b c} \chi^{d e} P_{b c, d e}=0 \\
\left\langle\tilde{\mathcal{L}}_{a} \mid T\right\rangle=2\left(G_{a b}-P_{a b}\right) B^{b}+N_{a}^{b c} \chi^{d e} P_{b c, d e}=0
\end{gathered}
$$

The solution of (C.18) is

$$
B^{a}=0, N_{a}^{b c} \chi^{d e} P_{b c, d e}=0 .
$$

Corollary 2: When $\chi^{a b} T_{a b}^{(-2)}|0\rangle \in V_{T}$ then $\chi^{a b} Q_{a b}{ }^{c}=0, \forall c$. This follows from Proposition 6 and the identity (A.14).

$\underline{\text { Proposition } 7} L^{(0)}$ maps $V_{\mathcal{L}}$ into itself and $V_{\tilde{\mathcal{L}}}$ into itself.

Proof: By explicit computation with (C.3), we obtain

$$
\begin{gathered}
L^{(0)}\left|\mathcal{L}_{a}\right\rangle=\left(\delta_{a}^{b}+M_{a}{ }^{b}\right)\left|\mathcal{L}_{b}\right\rangle \\
L^{(0)}\left|\tilde{\mathcal{L}}_{a}\right\rangle=M_{a}{ }^{b}\left|\tilde{\mathcal{L}}_{b}\right\rangle .
\end{gathered}
$$

because $T_{a b}^{(-1)}|0\rangle=0$.

Corollary 3: $L^{(0)}$ maps $V_{T}$ into itself.

Proof: Compute $\left\langle\mathcal{L}_{a}\left|L^{(0)}\right| T\right\rangle=\left\langle\tilde{\mathcal{L}}_{a}\left|L^{(0)}\right| T\right\rangle=0$ using Proposition 7 and the hermiticity of $L^{(0)}$. It follows that $L^{(0)}|T\rangle \in V_{T}$.

Proposition 8: Let $\left|\chi_{i}\right\rangle \equiv \chi_{i}^{a b} T_{a b}^{(-2)}|0\rangle$ be the eigenvectors of $L^{(0)}$ in $V_{T}$ with respective eigenvalue $\Delta_{i}$,

$$
L^{(0)} \chi_{i}^{a b} T_{a b}^{(-2)}|0\rangle=\Delta_{i} \chi_{i}^{a b} T_{a b}^{(-2)}|0\rangle, \quad i=1,2, \cdots, \operatorname{dim} V_{T}
$$

Then $\chi_{i}^{a b}$ is a right eigenvector of the stability matrix $\mathcal{S}$ with corresponding eigenvalue $1-\Delta_{i}$.

Proof: We compute $L^{(0)}\left|\chi_{i}\right\rangle$ using (C.3). The spin 3 terms proportional to $W$ and $X$ do not contribute because $W_{a b c}^{(-2)}|0\rangle=X_{a b}^{(-2)}|0\rangle=0$. According to corollary 2, the spin-1 terms proportional to $J$ do not contribute either because $\chi_{i}^{a b} Q_{a b}{ }^{c}=0 \forall i, c$. The result

$$
\chi_{i}^{a b} R_{a b}^{c d}=\Delta_{i} \chi_{i}^{c d} \forall i
$$

is then obtained by comparison with (C.21), and

$$
\mathcal{S}^{a b}{ }_{c d} \chi_{i}^{c d}=\left(1-\Delta_{i}\right) \chi_{i}^{a b}
$$

is obtained from (C.12).

Proposition 9: The eigenspace of $\mathcal{S}$ generated by $\chi_{i}^{a b} i=1,2, \cdots, \operatorname{dim} V_{T}$ is orthogonal to the eigenspace $V_{N}$ of $\mathcal{S}$ (see Prop. 3,4). 
Proof: Using Corollary 2 and the inner product (4.5a) in the right eigenspace of $\mathcal{S}$, compute

$$
\left\langle N_{a} \mid \chi_{i}\right\rangle=-N_{a}^{b c} P_{b c, d e} \chi_{i}^{d e}=-\chi_{i}^{d e} Q_{d e}^{b} G_{b a}=0
$$

Let $V_{\chi}$ be the eigenspace generated by $\chi_{i}^{a b} \forall i$, with $\operatorname{dim} V_{\chi}=\operatorname{dim} V_{T}$. Since $\operatorname{dim} V_{\chi}+\operatorname{dim} V_{N}=$ $D(D+1) / 2$, it follows that $V_{N} \oplus V_{\chi}$ is the space of right eigenvectors of $\mathcal{S}$. We have established the following

Theorem: $\mathcal{S}^{a b}{ }_{c d}$ has $D-N_{0}-N_{1}$ zero eigenvalues, with right eigenvectors in $V_{N}$. The other $N_{0}+N_{1}-D+D(D+1) / 2$ eigenvalues of $\mathcal{S}$ are given by $\left\{1-\Delta_{i}\right\}$ where $\left\{\Delta_{i}\right\}$ are the eigenvalues of $L^{(0)}$ in $V_{T}$.

Proposition 10: There exists an orthogonal decomposition $V_{T}=\operatorname{span}\{|L\rangle\} \oplus$ $\operatorname{span}|\tilde{L}\rangle \oplus V_{\perp}$, where $|L\rangle \equiv L^{(-2)}|0\rangle$ and $|\tilde{L}\rangle \equiv \tilde{L}^{(-2)}|0\rangle$. All the states in $V_{\perp}$ are primary with respect to the Virasoro generators $L^{(n)}$.

Proof: $\langle L \mid \tilde{L}\rangle=\left\langle 0\left|L^{(2)} \tilde{L}^{(-2)}\right| 0\right\rangle=\left\langle 0\left|\tilde{L}^{(-2)} L^{(2)}\right| 0\right\rangle=0$, so the decomposition is orthogonal. Each state $|\chi\rangle$ in $V_{\perp}$ satisfies $\langle L \mid \chi\rangle=\langle\tilde{L} \mid \chi\rangle=0$ which implies, according to (C.3), that $\chi^{a b} P_{a b}=\chi^{a b} G_{a b}=0$. Then

$$
\begin{gathered}
L^{(m>2)}|\chi\rangle=0, L^{(2)}|\chi\rangle=\chi^{a b} P_{a b}|0\rangle=0 \\
L^{(1)}|\chi\rangle=\frac{3}{2} \chi^{a b} R_{a b}{ }^{c d} T_{c d}^{(-1)}|0\rangle=0
\end{gathered}
$$

also follows by (C.3) for each state in $V_{T}$.

\section{References}

[1] V.G. Kač, Funct. Anal. App. 1 (1967) 328; R.V. Moody, Bull. Am. Math. Soc. 73 (1967) 217.

[2] K. Bardacki and M.B. Halpern, Phys. Rev. D3 (1971) 2493.

[3] P. Ramond, Phys. Rev. D3 (1971) 2415.

[4] M.B. Halpern, Phys. Rev. D4 (1971) 2398.

[5] M.B. Halpern, Phys. Rev. D12 (1975) 1684.

[6] T. Banks, D. Horn and H. Neuberger, Nucl. Phys. B108 (1976) 119.

[7] I.B. Frenkel and V.G. Kač, Inv. Math. $\underline{62}$ (1980) 23; G. Segal, Comm. Math. Phys. $\underline{80}$ (1981) 301.

[8] E. Witten, Comm. Math. Phys. $\underline{92}$ (1984) 455; G. Segal, unpublished; V.G. Knizhnik and A.M. Zamolodchikov, Nucl. Phys. B247 (1984) 83. 
[9] P. Goddard, A. Kent and D. Olive, Phys. Lett. B152 (1985) 88.

[10] D. Gross, J.A. Harvey, E. Martinec and R. Rohm, Phys. Rev. Lett. 54 (1985) 502.

[11] J.K. Freericks and M.B. Halpern, Ann. of Phys. 188 (1988) 258; Erratum, ibid. 190 (1989) 212.

[12] M.B. Halpern, Ann. of Phys. 194 (1989) 247.

[13] P. Goddard and D. Olive, Int. J. Mod. Phys. A1 (1986) 303.

[14] I.B. Frenkel, J. Lepowsky and A. Meurman, to appear.

[15] M.B. Halpern and E. Kiritsis, Mod. Phys. Lett. A4 (1989) 1373; Erratum ibid. A4 (1989) 1797.

[16] M.B. Halpern and J.P. Yamron, Nucl. Phys. B332 (1990) 411.

[17] A.Yu Morozov, A.M. Perelomov, A.A. Rosly, M.A. Shifman and A.V. Turbiner, Int. J. Mod. Phys. A5 (1990) 803.

[18] E. Kiritsis, Mod. Phys. Lett. A4 (1989) 437; G. V. Dunne, I. G. Halliday and P. Suranyi, Phys. Lett. B213 (1988) 139.

[19] M.B. Halpern, E. Kiritsis, N.A. Obers, M. Porrati and J.P. Yamron, Int. J. Mod. Phys. A5 (1990) 2275.

[20] E. Witten, in Memorial Volume for V. Knizhnik, ed. L. Brink et al., World Scientific, 1990.

[21] M.B. Halpern and N.A. Obers, "Unitary Irrational Central Charge on Compact g. I. (Simply-Laced $\left.g_{x}\right)_{\#}^{q \geq 2 "}$, Berkeley preprint, UCB-PTH-89/35, (1989). To appear in Int. J. Mod. Phys. A

[22] S. Schrans and W. Troost, Nucl. Phys. B345 (1990) 584.

[23] M.B. Halpern and N.A. Obers, Nucl. Phys. B345 (1990) 607.

[24] M.B. Halpern and N.A. Obers, "Graph Theory, SO(n) Current Algebra and the Virasoro Master Equation", Berkeley preprint, UCB-PTH-90/31, (1990).

[25] A. Yu Morozov, M.A. Shifman and A.V. Turbiner, Int. J. Mod. Phys. A5 (1990) 2953.

[26] F. Harary and E.M. Palmer, Graphical Enumeration. New York, London, Academic Press 1973. 
[27] M.B. Halpern and J.P. Yamron, "A Generic Affine-Virasoro Action", Berkeley preprint, UCB-PTH-90/22 (1990). To appear in Nucl. Phys. B

[28] N. Mohammedi, "General Super Virasoro Construction on Affine g", Trieste preprint, PRINT-90-0645 (1990)

[29] A.B. Zamolodchikov, JETP. Lett. $\underline{43}$ (1986) 730; A.A. Tseytlin, Phys. Lett. B194 (1987) 63; J. Cardy, Phys. Rev. Lett. 60 (1988) 2709; A. Capelli, D. Friedan and J. Latorre, "C-Theorem and Spectral Representation", Rutgers preprint, RU-90-43 (1990)

[30] M.B. Halpern and N.A. Obers, in preparation

[31] V. G. Kač, "Infinite dimensional Lie algebras", Cambridge Univ. Press, 1985.

[32] L. Dixon, M. E. Peskin and J. Lykken, Nucl. Phys. B325 (1989) 329.

[33] M.B. Halpern, E.B. Kiritsis and N.A. Obers, in preparation

[34] C. Vafa, Phys. Lett. B212 (1988) 28.

[35] S.R. Das, G. Mandal and S. Wadia, Mod. Phys. Lett. A4 (1989) 745

[36] E. Witten, Nucl. Phys. B188 (1981) 513.

[37] D. Friedan as quoted in T. Banks and E. Martinec, Nucl. Phys. B293 (1987) 733.

[38] M. Claudson and M.B. Halpern, Ann. of Phys. 166 (1986) 33

[39] J. Greensite and M.B. Halpern, Nucl. Phys. B242 (1984) 167; E. Marinari and G. Parisi, Phys. Lett. B240 (1990) 375; M. Karliner and A. Migdal, "Non-perturbative 2D Quantum Gravity via Supersymmetric String", Princeton preprint, PUPT-1191, (1990).

[40] M. Claudson and M.B. Halpern, Phys. Lett. B151 (1985) 281; Z. Bern and H.S. Chan, Nucl. Phys. B266 (1986) 509. 